Check for updates

Cite this: Phys. Chem. Chem. Phys., 2020, 22, 21707

Received 10th June 2020,

Accepted 14th September 2020

DOI: $10.1039 / d 0 c p 03105 d$

rsc.li/pccp

\section{Accessing distributions of exchange and dipolar couplings in stiff molecular rulers with $\mathrm{Cu}(\mathrm{II})$ centres $\dagger$}

\author{
K. Keller, ${ }^{a}$ I. Ritsch, ${ }^{a}$ H. Hintz, ${ }^{b}$ M. Hülsmann, ${ }^{b}$ M. Qi, ${ }^{b}$ F. D. Breitgoff, ${ }^{a}$ D. Klose, ${ }^{a}$ \\ Y. Polyhach, (D) ${ }^{\star a}$ M. Yulikov, (DD ${ }^{* a} A$. Godt ${ }^{* b}$ and G. Jeschke (D) ${ }^{a}$
}

\begin{abstract}
Determining distributed exchange couplings is important for understanding the properties of synthetic magnetic molecules. Such distributions can be determined from pulsed dipolar spectroscopy (PDS) data, but this is challenging due to the similar influence of both exchange and dipolar couplings on such data. In this work we introduce two models that aim to identify these two contributions to the spin-spin couplings from frequency-domain PDS data of shape-persistent molecules having either two Cu(II) ions, or a $\mathrm{Cu}(॥)$ ion and a nitroxide radical as the paramagnetic moieties. The first model assumes correlated Lorentzian or Gaussian exchange and dipole-dipole coupling distributions whose parameters are the model's unknowns. The second model relies on prior knowledge of the distance distribution and by performing Tikhonov regularization along the exchange coupling dimension yields the latter distribution model-free. Both models were able to differentiate between the absence and the presence of exchange interaction, to determine the coupling regime (ferro- or antiferromagnetic) and to estimate the distribution shape. In contrast, calculations within the exchange resilient model of the neural network analysis implemented in DeerAnalysis2018 were not able for our data to identify exchange couplings and return correct distance distributions. However, the generic model was able to identify and separate the strongly curved intermolecular background in the relaxation-induced dipolar modulation enhancement (RIDME) experiments. Our analysis revealed that in such systems exchange coupling may be present up to at least $3.3 \mathrm{~nm}$ in $\pi$-conjugated systems involving $\mathrm{Cu}(I)$-PyMTA, while it is negligible for distances $r \geq 4.5 \mathrm{~nm}$ between $\mathrm{Cu}(॥)$ ions and $r \geq 3.8 \mathrm{~nm}$ between a $\mathrm{Cu}(॥)$ ion and an unpaired electron of a nitroxide radical. Disruption of the $\pi$-conjugation between the ligand of the $\mathrm{Cu}(\Perp)$ complex and the nitroxide leads to negligible exchange coupling at distances $r \geq 2.6 \mathrm{~nm}$ in the corresponding $\left[\mathrm{Cu}\left({ }_{1}\right)-\mathrm{TAHA}\right]-$ nitroxide ruler. Overall, for cases with known distance distributions, the presented analysis techniques allow to determine distributions of exchange couplings from PDS data.
\end{abstract}

\section{Introduction}

Electron paramagnetic resonance (EPR) spectroscopy is a valuable tool in the study of macromolecular assemblies. Pulse dipolar EPR spectroscopy (PDS) makes it possible to specifically probe magnetic spin-spin couplings between paramagnetic centers. Such a coupling has two main contributions: the dipole-dipole and the exchange interaction. ${ }^{1-3}$ In biomolecular studies PDS

\footnotetext{
${ }^{a}$ Laboratory of Physical Chemistry, Department of Chemistry and Applied Biosciences, ETH Zurich, Vladimir-Prelog-Weg 2, 8093 Zurich, Switzerland. E-mail: yevhen.polyhach@phys.chem.ethz.ch, maxim.yulikov@phys.chem.ethz.ch

${ }^{b}$ Faculty of Chemistry and Center for Molecular Materials $\left(\mathrm{CM}_{2}\right)$, Bielefeld

University, Universitätsstraße 25, 33615 Bielefeld, Germany.

E-mail: godt@uni-bielefeld.de

$\dagger$ Electronic supplementary information (ESI) available. See DOI: 10.1039/ d0cp03105d
}

experiments are primarily used to determine distance restraints. Therefore, whenever possible, spin pairs are designed such that only the dipole-dipole interaction plays a significant role in the spin-spin coupling, which can readily be interpreted in terms of inter-spin distance distributions. ${ }^{1,4-6}$ In contrast to biomolecules, synthetic organic molecules are often designed to have specific magnetic properties in order to be used as molecular magnets, ${ }^{7,8}$ DNP enhancers, ${ }^{9}$ NMR/MRI contrast agents, ${ }^{10-13}$ or for studies on quantum information processing. ${ }^{14-16}$ This benefits from the design of spin pairs with tunable strengths of dipole-dipole as well as exchange interactions between electron spins.

Paramagnetic $\mathrm{Cu}$ (II) ions are interesting for determining distances between specific sites, either naturally occurring metal binding sites of metalloproteins ${ }^{17-20}$ or selected sites of interest, where $\mathrm{Cu}$ (II) spin $\operatorname{tags}^{21-23}$ can be attached by sitedirected spin labelling. ${ }^{24-27}$ Significant progress in performing 
and analysing $\mathrm{Cu}$ (II)-based PDS experiments has been achieved over the past few years. ${ }^{28-33}$ Exchange interactions are often observed for $\mathrm{Cu}$ (II) ions that are bound to conjugated $\pi$-systems, ${ }^{34-39}$ or in strongly coupled systems, such as molecular magnets. ${ }^{7,40,41}$ The exchange coupling may give additional information on the system of interest, yet, the dipolar and exchange couplings can become difficult to disentangle. . $^{3,42-44}$

Approaches to determine exchange coupling strengths based on the analysis of the EPR lineshape have been reported. ${ }^{45-48}$ In cases, where the isotropic tumbling limit is achieved in liquid solution, the separation of isotropic exchange and anisotropic dipole-dipole coupling is straightforward because anisotropic contributions are averaged to zero as for example demonstrated for $\mathrm{Cu}(\mathrm{II})$ compounds in ref. 34 and 35 . Note however that fast dynamics of the molecules in solution averages distribution of the exchange couplings for different possible conformations of the magnetic molecule as well as for dynamical shape distortions within each conformer, so that only the mean value can be accessed in this regime.

In the other limiting case, i.e. in the rigid limit, in which molecules are randomly oriented and immobilized in a glassy solution, both exchange and dipole-dipole couplings influence the shape of the EPR spectrum. If the half-field transition which is independent from isotropic exchange coupling - can be detected, it may be a convenient measure to separate the exchange and dipolar contributions. ${ }^{42,49}$ Generally, separating and accurately determining both couplings in EPR lineshape analysis is a complex task. All parameters must be explicitly taken into account which influence the resonance frequencies of the coupled spins, such as principal values and relative orientations of all relevant magnetic tensors ( $g$-tensor, hyperfine coupling tensor etc.). The problem is complicated further if any of the parameters in question are distributed. In such cases, independent knowledge of the molecular structure is helpful as it can, for instance, provide information on the dipole-dipole couplings, and leave only the distribution of exchange couplings as an unknown. ${ }^{39}$ Finally, if the line width exceeds the spin-spin coupling strength, the latter remains unresolved in EPR spectra in both the fast tumbling and the rigid limit regimes, thus making the EPR lineshape analysis approach inapplicable for determining spin-spin interactions.

For many spin systems the spin-spin couplings can be separated well from other interactions, such as $g$ and hyperfine anisotropies by PDS. Hence, knowledge of the principal values of the corresponding magnetic tensors as well as their mutual orientations is no longer strictly required to be able to analyze the spin-spin couplings. Another strength of PDS experiments is that they can provide detailed information on the distribution of spin-spin couplings. For instance, in biological applications this is used to obtain site-to-site distance distributions from precisely measured dipole-dipole coupling distributions., ${ }^{1,-6}$ In synthetic magnetic molecules it is also important to understand how broadly the spin-spin couplings are distributed. Such magnetic molecules are often designed to have a fixed geometry, in order to obtain defined magnetic properties. This implies a rather narrow distribution of spin-spin distances, and, hence, dipolar couplings. However, since the exchange interaction strongly depends on the degree of orbital overlap, it can vary substantially even upon small distortions of the molecule structure. Thus, while for such magnetic molecules the assumption of narrow spin-spin distance distribution is often valid, the distribution of exchange couplings could still be quite broad. ${ }^{36,37}$ Furthermore, stiffness of magnetic molecules facilitates the so called orientation-selection effect, ${ }^{50}$ where, depending on the excitation scheme of a particular experiment (e.g. microwave pulse positions, frequencies, excitation bandwidths and shape of the pulses etc.) different parts of the overall spin-spin coupling distribution can be attenuated or amplified reflecting the molecular geometry. Thus, orientation selection data are richer in information. However, processing of such data is much more complicated as it requires to consider orientations of hyperfine and $g$ tensors.

In some practical cases, e.g. molecular magnets or certain types of biradicals used for DNP, the dipolar and exchange coupling values may be too large to be detected with PDS. However, measurements of weaker spin-spin couplings on analogous magnetic molecules appear useful for understanding the principles of the magnetic molecule design. Spin-spin coupling can be controlled by varying the distance between the two paramagnetic moieties in the molecule. ${ }^{51}$

PDS experiments are usually performed in frozen glassy solvents and, thus, they can reveal distributions of exchange couplings for different conformations of the magnetic molecules, representing a snapshot of the dynamic processes in solution corresponding to the glass transition temperature. ${ }^{52}$ Such a determination in PDS experiments is, however, still quite challenging due to the mentioned combination of distributions of dipole-dipole and exchange couplings along with orientation selection effects. Quite substantial work was done to demonstrate how orientation selection effects in the PDS experiments can be reduced by averaging over a set of pump and detection positions in $\mathrm{Cu}(\mathrm{II})$ and nitroxide EPR spectra. ${ }^{29,33,53-56}$ Such (partially) orientation-averaged PDS data are good test examples to study whether and how accurately the distributions of exchange and dipolar couplings can be disentangled and simultaneously determined. In the most general case, where both dipolar and exchange couplings are broadly distributed and correlated to each other, information content of the data may be too small for unambiguous analysis. However, many practical examples allow for simplifications, which then make the overall analysis more reliable.

Any PDS approach relies on the detection of time traces that oscillate with frequencies determined by the spin-spin coupling distribution. It is limited by the transverse relaxation time and thus exhibits higher resolution than the inhomogeneously broadened EPR spectrum. Fourier transform of such time traces yields an exchange-distorted Pake-like dipolar frequency pattern. If its singularities are well resolved, their dependence on exchange and dipolar interaction ${ }^{57,58}$ can be used to extract the individual couplings. ${ }^{59}$ Extraction of spin-spin couplings has also been performed by simulation of the experimental form factor and taking the spin-spin couplings as well as excited orientations 
into account. ${ }^{36,37,60,61}$ In some of the cases singularities of the Pake-like patterns were taken as initial guess for the spin-spin couplings in the simulation of the full time traces. Similar simulations, with an analytical expression of the spin-spin couplings in time domain, have been presented for time traces with negligible orientation-selection and presuming that the spin-spin distance is known. ${ }^{38}$

We have recently encountered a curious case of exchange interaction in shape-persistent molecules featuring $\mathrm{Cu}$ (II)-Cu(II) or $\mathrm{Cu}(\mathrm{II})$-nitroxide radical spin pairs, wherein the strength of spin-spin exchange interaction depends on the types of paramagnetic moieties involved. In case of the $\mathrm{Cu}$ (II) ion complex, it also depends on the type of ligand. In this contribution, we thus address the problem of determining exchange coupling and distance distributions with four different shape-persistent molecules involving $\mathrm{Cu}(\mathrm{II})$ ions (see Fig. 1). We apply the chelators PyMTA or TAHA and consider $\mathrm{Cu}$ (II)-Cu(II) as well as $\mathrm{Cu}(\mathrm{II})$-nitroxide rulers with both types of chelators. We attempt to extract electron spin-spin couplings from orientation-averaged frequency-domain PDS data using three different approaches.
The paper is structured as follows: first, we describe the studied spin systems, their synthesis and their characterization by CW EPR and DFT calculations. Second, we present UV/VIS absorption spectra for the ruler compounds and the corresponding ruler precursors before $\mathrm{Cu}(\mathrm{II})$-loading. Next, we describe the time traces obtained by Relaxation-Induced Dipolar Modulation Enhancement (RIDME) ${ }^{62,63}$ and Double Electron Electron Resonance (DEER) ${ }^{64,65}$ experiments on these compounds. Fourth, on the example of the [Cu-PyMTA $]-[\mathrm{Cu}-\mathrm{PyMTA}]$ ruler, we test the applicability of our analysis routines and of analysis by neural networks as available in DeerAnaylsis2018. ${ }^{66}$ Finally, we apply the best performing data analysis to all other studied systems.

\section{Theoretical background}

The magnetic interactions are described by the system's spin Hamiltonian. For the $\mathrm{Cu}(\mathrm{II})-\mathrm{Cu}(\mathrm{II})$ and $\mathrm{Cu}(\mathrm{II})$-nitroxide rulers studied in this work, the following spin Hamiltonian was

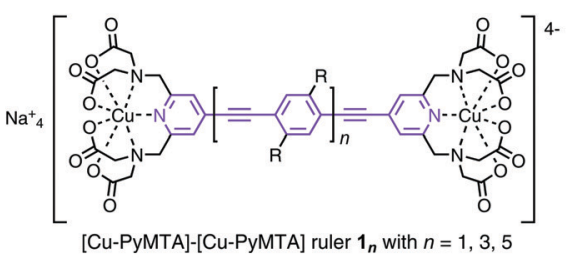

[Cu-PyMTA]-[Cu-PyMTA] ruler $\mathbf{1}_{n}$ with $n=1,3,5$

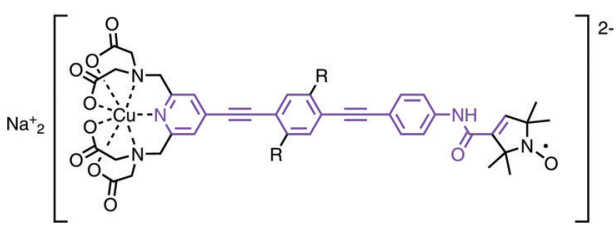

[Cu-PyMTA]-nitroxide ruler $\mathbf{2}_{1}$

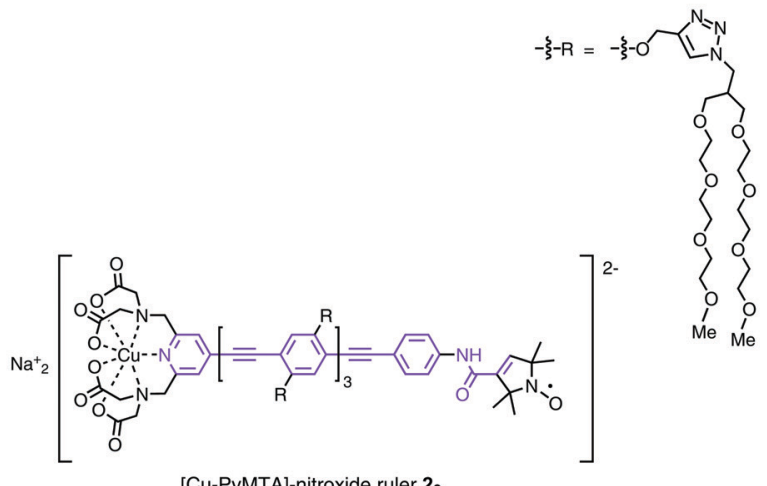

[Cu-PyMTA]-nitroxide ruler $\mathbf{2}_{\mathbf{3}}$

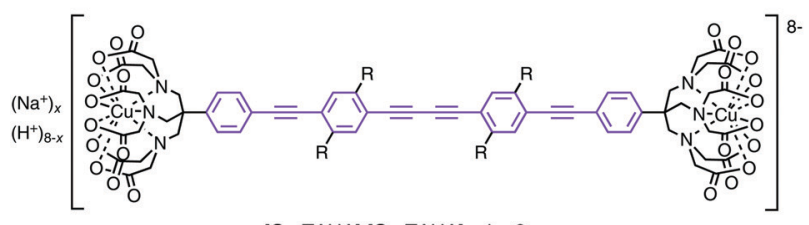

[CU-TAHA]-[CU-TAHA] ruler 3

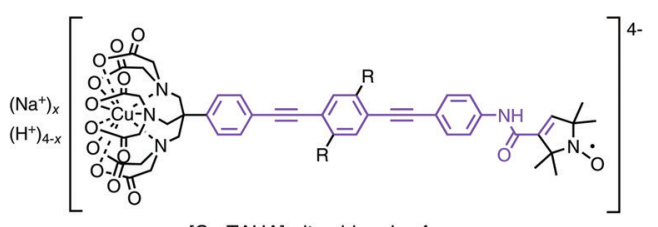

[CU-TAHA]-nitroxide ruler 4

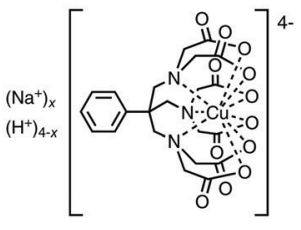

CU-TAHA

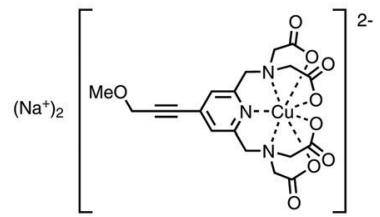

Cu-PyMTA

Fig. 1 Structural formulae of the $\mathrm{Cu}(\|)-\mathrm{Cu}(\|)$ and $\mathrm{Cu}(\|)$ - nitroxide rulers and the corresponding complexes $\mathrm{Cu}(\|)-\mathrm{PyMTA}$ and $\mathrm{Cu}(\|)-\mathrm{TAHA}$ used in this study. The effective spacer is marked violet. The displayed coordination of the $\mathrm{Cu}(I)$ ions shows the maximum potential coordination bonds. See Section 4.1 for the question about the numbers of coordinating atoms. The Cu-TAHA moiety is partially protonated with about 1.9 protons per moiety. ${ }^{56}$ Due to the structural similarity of PyMTA to diethylenetriaminepentaacetic acid (DTPA) the Cu-PyMTA moiety is expected to be completely deprotonated at $\mathrm{pH} 7$ as reported for the complex $\mathrm{Cu}(I)-$ DTPA. ${ }^{75}$ 
considered for two coupled electron spins A and B forming a spin pair:

$$
\begin{aligned}
\hat{H}= & \frac{\mu_{\mathrm{B}}}{\hbar} \vec{B}_{0} \mathbf{g}_{\mathrm{A}} \overrightarrow{\hat{S}}_{\mathrm{A}}+\frac{\mu_{\mathrm{B}}}{\hbar} \vec{B}_{0} \mathbf{g}_{\mathrm{B}} \overrightarrow{\hat{S}}_{\mathrm{B}}+\sum_{i} \overrightarrow{\hat{\hat{S}}}_{\mathrm{A}} \mathbf{A}_{\mathrm{A}, i} \overrightarrow{\hat{I}}_{i} \\
& +\sum_{i} \overrightarrow{\hat{S}}_{\mathrm{B}} \mathbf{A}_{\mathrm{B}, i} \overrightarrow{\hat{I}}_{i}+\overrightarrow{\hat{S}}_{\mathrm{A}}(J+\mathbf{D}) \overrightarrow{\hat{S}}_{\mathrm{B}}
\end{aligned}
$$

Here $\mu_{\mathrm{B}}$ is the Bohr magneton, $\hbar$ the reduced Planck constant, $\mathbf{g}_{\mathrm{A} / \mathrm{B}}$ are the $g$-tensors of the two electron spins A and $\mathrm{B}, \mathbf{A}_{i}$ is the hyperfine tensor, $J$ the exchange coupling constant, and $\mathbf{D}$ the dipole-dipole coupling tensor.

The first two terms describe the orientation-dependent electron Zeeman interaction for the A and B spins. The next two terms describe the hyperfine interaction between the electron spins and non-zero nuclear spins. The last term describes electron-electron spin interactions $\hat{H}_{\text {ee }}$. In the weak coupling approximation, which applies to an overwhelming majority of spin pairs because of the strong anisotropy of the $g$ and $\mathrm{Cu}(\mathrm{II})$ hyperfine tensors and relatively small spin-spin coupling strengths, this term can be simplified to:

$$
\hat{H}_{\mathrm{ee}}=J \hat{S}_{\mathrm{A}, z} \hat{S}_{\mathrm{B}, z}+\omega_{\mathrm{dd}} \hat{S}_{\mathrm{A}, z} \hat{S}_{\mathrm{B}, z}
$$

The dipolar coupling $\omega_{\mathrm{dd}}$ is dependent on the angle $\theta$ between the spin-spin vector and the external magnetic field

$$
\omega_{\mathrm{dd}}=\frac{\mu_{0} \mu_{\mathrm{B}}^{2} g_{\mathrm{A}, \mathrm{eff}} g_{\mathrm{B}, \mathrm{eff}}}{4 \pi \hbar r_{\mathrm{AB}}{ }^{3}}\left(1-3 \cos ^{2} \theta\right) .
$$

It is also dependent on the effective $g$-value of each spin at a given orientation $(\phi, \theta)$

$$
g_{\mathrm{eff}}(\phi, \theta)=\sqrt{g_{x}^{2} \sin ^{2} \theta \cos ^{2} \phi+g_{y}^{2} \sin ^{2} \theta \sin ^{2} \phi+g_{z}^{2} \cos ^{2} \theta},
$$

which is described by the electron Zeeman Hamiltonian transformed to its eigenframe

$$
\hat{H}_{\mathrm{EZ}}=\frac{\mu_{\mathrm{B}}}{\hbar} g_{\mathrm{eff}} B_{0} \hat{S}_{z} .
$$

$g_{x}, g_{y}$, and $g_{z}$ are the principal values of the $g$ tensor and the polar angles $\theta$ and $\phi$ determine the orientation of the magnetic field in the principal axis system.

The dependency of the dipolar coupling constant on the angle $\theta$ between the spin-spin axis and the external magnetic field can lead to aforementioned orientation selectivity effects in experimental dipolar evolution data if not all orientations are excited uniformly. ${ }^{50}$ Such data can be described by introducing a geometry factor $\xi(\theta)$, which accounts for excited fractions of orientations in the signal evolution and depends on sample geometry and the applied microwave pulses setup: ${ }^{58}$

$$
V(t)=\iint P\left(r_{\mathrm{AB}}\right) \xi(\theta) \cos \left(\left[\omega_{\mathrm{dd}}\left(r_{\mathrm{AB}}, \theta\right)+J\right] t\right) \mathrm{d} \theta \mathrm{d} r_{\mathrm{AB}}
$$

In cases where all orientations can be excited uniformly, or the time-domain signal can be completely averaged over all orientations, the signal in frequency domain corresponds to the Pake-like pattern for a given spin-spin distance $r_{\mathrm{AB}}$ and exchange coupling constant $J .^{57,58}$ In fact, orientation selectivity effects are often observed as distortions of the Pake-like pattern and they may complicate data analysis significantly as knowledge of the molecular geometry is required in such cases. The strong anisotropy of $\mathrm{Cu}$ (II) spectra and insufficient excitation bandwidth of microwave pulses often lead to orientation selection effects. ${ }^{36,54,67-70}$ Such effects can be included into data analysis and give additional information on the geometry of the system. ${ }^{36,69,70}$ However, to simplify the problem of disentangling exchange and dipole-dipole couplings, we aimed to acquire data with negligible orientation selection by averaging over several detection fields. ${ }^{33,53-55}$

Extraction of the spin-spin interactions between two paramagnetic centres with broad EPR spectra posed the need to develop PDS experiments that can cover a large bandwidth. In recent years two main techniques developed in this respect: the use of ultrawideband (UWB) inversion pulses in combination with the DEER experiment ${ }^{71}$ or inversion by stochastic longitudinal relaxation events instead of an inversion pulse in the RIDME sequence. ${ }^{62,63,72}$

The (UWB-)DEER and RIDME pulse sequences are shown in Fig. 2. In both experiments electron spin coherence is created by the first $\pi / 2$ pulse of the initial preparation period $(\pi / 2-\tau-\pi$ sequence). After this initial preparation period, a modulation to the local field at the position of the observed spin A is induced by inversion of a second spin B. This causes a shift of the resonance frequency of the A spin. The change in local field is proportional to the spin-spin coupling, which in turn depends on the inverse cube of the A-B distance and on orientation for the dipole-dipole coupling, as well as on the exchange coupling constant. Thus, depending on the inversion efficiency $p_{\text {inv }}$ of the coupled B spin, a fraction of the electron spin echo signal will oscillate with the sum of the dipolar frequency $\omega_{\mathrm{dd}}$ and the exchange coupling constant $J$. This fraction $\lambda$ is often called modulation depth. The evolution of the A spin with changed
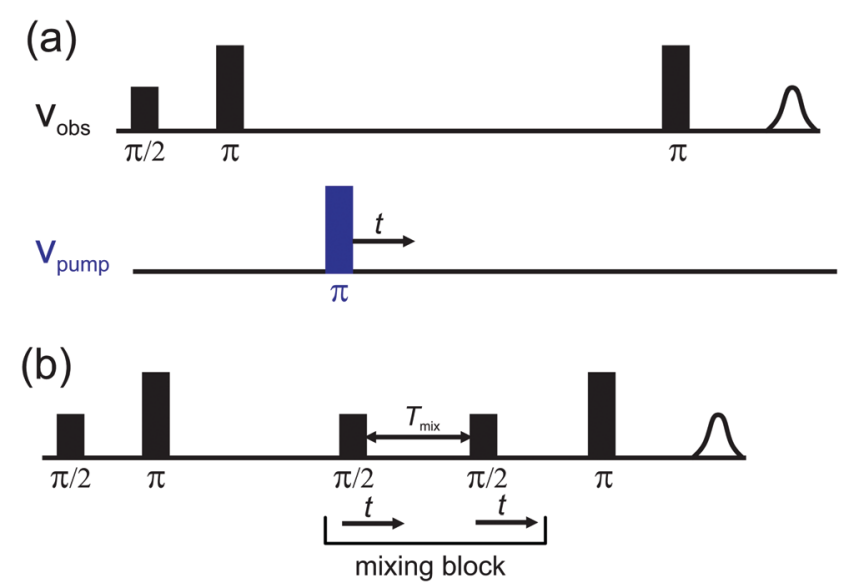

Fig. 2 (a) Four-pulse DEER ${ }^{65}$ and (b) five-pulse RIDME ${ }^{63}$ sequences. In (a) the dipolar evolution signal is detected as the intensity variation of the refocused primary echo with respect to the shift of the time position of the pump pulse (blue). In (b) the so-called refocused virtual echo is detected (see ref. 63) and its intensity varies with the change of the position of the mixing block, formed by the last two $\pi / 2$ pulses. 
frequency results in a phase gain of $\pm\left(\omega_{\mathrm{dd}}+J\right) t$, which is then detected using a final refocusing pulse. An important difference between the two pulse sequences is the nature of the inversion of the B spins.

In the DEER experiment the B spins are inverted by a pump pulse applied at a second frequency (or with a frequencymodulated pulse, whose excitation band does not overlap with the detection band) and the inversion efficiency is given by the excitation profile of the pump pulse. In the absence of orientation selection, the modulation depth of the DEER experiment is equal to the inversion efficiency of the pump pulse.

In the RIDME experiment the magnetization is stored in the direction of the external field in the form of a polarization grating for a certain time $T_{\text {mix }}$. During this mixing time $T_{\text {mix }}$ longitudinal relaxation events lead to stochastic inversion of $\mathrm{B}$ spins. After the mixing time, the magnetization is transferred back into the transverse plane, where it is eventually detected. The modulation depth is thus determined by the time allowed for such B spin flips. To first approximation, the modulation builds up according to the probability of an odd number of spin flips during the mixing block: ${ }^{73,74}$

$$
P_{\text {odd }}=\frac{1}{2}\left(1-\exp \left(-T_{\text {mix }} / T_{1, \mathrm{~B}}\right)\right) \text {. }
$$

\section{Experimental and computational details}

\subsection{Synthesis details}

The structural formulae of the compounds used in this study are shown in Fig. 1 . The syntheses of rulers $2_{1}$, and $4^{56}$ and of reference mono-complex compounds $\mathrm{Cu}-\mathrm{PyMTA}^{74}$ and $\mathrm{Cu}-\mathrm{TAHA}^{56}$ have been described previously. The syntheses of the rulers $2_{3}$ and 3 are described and discussed below (Section 4.1). The details for the syntheses of rulers $\mathbf{1}_{n}, \mathbf{2}_{3}$, and $\mathbf{3}$ are given in the Supporting information part 2 (ESI $2 \dagger)$.

\subsection{EPR sample preparation}

Stock solutions of all compounds in $\mathrm{D}_{2} \mathrm{O}$ were diluted in a $1: 1$ $(\mathrm{v}: \mathrm{v}) \mathrm{D}_{2} \mathrm{O} /$ glycerol- $\mathrm{d}_{8}$ mixture to a final concentration of $200 \mu \mathrm{M}$. CW EPR samples for Cu-PyMTA and Cu-TAHA (reference mono-complex compounds) were diluted to a final concentration of $1 \mathrm{mM}$ in a $1: 1(\mathrm{v}: \mathrm{v}) \mathrm{H}_{2} \mathrm{O} /$ glycerol mixture. The sample solutions were filled into $1.6 \mathrm{~mm}$ or $3 \mathrm{~mm}$ o.d. quartz capillaries depending on the resonator used and subsequently flash-frozen by immersion into liquid nitrogen.

\subsection{EPR measurements}

CW X-band EPR spectra were detected on a Bruker Elexsys E500 spectrometer equipped with a Bruker Elexsys Super High Quality probehead and a nitrogen flow cryostat. Measurements were performed at $140 \mathrm{~K}, 100 \mathrm{kHz}$ field modulation, $0.4 \mathrm{mT}$ modulation amplitude, $1400 \mathrm{mT}$ field sweep, and $0.2 \mathrm{~mW}$ microwave power (30 dB attenuation on the spectrometer used). The time constant was $40.96 \mathrm{~ms}$ and the conversion time $327.68 \mathrm{~ms}$.
Q-band data were acquired either on a commercial Bruker Elexsys E580 Q-band spectrometer or on a home-built spectrometer based on a Keysight Arbitrary Waveform Generator $(\mathrm{AWG})^{76}$ with a high-power Q-band extension. ${ }^{77}$ A helium flow cryostat (ER $4118 \mathrm{CF}$, Oxford Instruments) was used to adjust the measurement temperature to $20 \mathrm{~K}$. The commercial spectrometer, equipped with a home-built cavity operating at about $34.5 \mathrm{GHz},{ }^{78,79}$ was used for the metal-metal RIDME experiments. The home-built spectrometer was combined with either the same resonator for the orthogonal-RIDME experiments or with a broadband resonator with a pent loop-gap $\operatorname{design}^{80}$ for the DEER experiments. For the pent loop-gap resonator the coupler position was set to provide the lowest possible $Q$-value, which was previously estimated to be about 50 , corresponding to a bandwidth of about $700 \mathrm{MHz}{ }^{80}$

$\mathrm{Cu}(\mathrm{II})-\mathrm{Cu}$ (II) RIDME data were acquired at $20 \mathrm{~K}$ using the refocused five-pulse sequence shown in Fig. 2(a) with $\pi / 2$-pulses set to $12 \mathrm{~ns}$ and $\pi$-pulses to $24 \mathrm{~ns}$. The sequence was $\frac{\pi}{2}-d_{1}-$ $\pi-\left(d_{1}+t\right)-\frac{\pi}{2}-T_{\text {mix }}-\frac{\pi}{2}-\left(d_{2}-t\right)-\pi_{\mathrm{obs}}-d_{2}-$ echo. The initial time $t$ was set to $d_{3,0}-d_{1}$, with $d_{1}=400 \mathrm{~ns}$ and $d_{3,0}=280 \mathrm{~ns}$ (negative initial $t=-120 \mathrm{~ns}$ before the position of the primary echo), in order to measure both sides of the region around zero dipolar evolution time. The interpulse delay $d_{2}$ was adjusted for each sample. If not stated otherwise, the mixing time was set to $T_{\text {mix }}=40 \mu$ s for the ruler $\mathbf{1}_{3}, \mathbf{1}_{5}$ and 3 . To remove echo crossing and phase offsets, an eight-step phase cycle was used. ${ }^{63}$ ESEEM averaging $^{81}$ was performed. To reduce orientation-selection effects, RIDME data were acquired at different observer fields $s^{29,33,53-55}$ over the full $\mathrm{Cu}$ (II) spectrum using a spacing of 150 G. Resulting time traces were normalized to unit intensity at the RIDME zero-time and summed weighted by the spectral intensity of each observer position. With this approach most observer orientations are expected to be excited uniformly. Orientation selection from the inverted spin is assumed to be negligible in RIDME experiments because relaxation events can occur in the full spectral range.

$\mathrm{Cu}(\mathrm{II})-\mathrm{Cu}$ (II) DEER data were recorded at $20 \mathrm{~K}$ with the deadtime free 4-pulse DEER sequence $\frac{\pi}{2}{ }_{\text {obs }}-\tau_{1}-\pi_{\mathrm{obs}}-\left(\tau_{1}+t\right)-$ $\pi_{\text {pump }}-\left(\tau_{2}-t\right)-\pi_{\text {obs }}-\tau_{2}-$ echo. ${ }^{65}$ The delay $\tau_{1}$ was set to $400 \mathrm{~ns}$ and $\tau_{2}$ was adjusted for the different compounds. All observer pulses were $12 \mathrm{~ns}$ long and set $100 \mathrm{MHz}$ off the center of the resonator mode $f_{0}$. The pump pulse was a sech/tanh (HS) pulse of order $6 .^{82,83}$ The length was $100 \mathrm{~ns}$, the bandwidth $800 \mathrm{MHz}$ and the frequency flank facing the observer pulse was offset from the observer frequency by $100 \mathrm{MHz} .^{33} \mathrm{HS}$ pulses were compensated for the resonator profile and non-linearity of the excitation chain. $^{76}$ Two pulse setup versions were employed, one in which the observer pulses were set to higher frequency than the pump pulse, and one for which the observer pulses were lower in frequency. Similar to the RIDME experiments, measurements were performed with the observer pulses at the maximum of the field swept spectrum and at several offsets from the maximum in steps of $150 \mathrm{G}$, respectively. The DEER traces from the individual field positions were added corresponding to the 
spectral intensity of each observer position. Finally, the summed traces from both setups were normalized and added together. With such an averaging procedure, RIDME and DEER traces agree sufficiently well to indicate (see Supporting information part 1 (ESI $1 \dagger)$ ) that orientation selection effects are strongly suppressed.

$\mathrm{Cu}(\mathrm{II})$-nitroxide RIDME data were acquired at $20 \mathrm{~K}$ using the resonance offset-compensated five-pulse RIDME sequence ( $2: 2: 2: 2: 1$ length ratio of the frequency-swept pulses) following the previously reported procedure. ${ }^{56}$ To cope with orientationselection effects the pulses had a bandwidth of $400 \mathrm{MHz}$, which was centered within the nitroxide spectrum. The $\pi / 2$-pulses and the long $\pi$-pulse were 150 ns long, the short $\pi$-pulse was $75 \mathrm{~ns}$ long. Quarter-sine flanks were used with rise-times of $30 \mathrm{~ns}$ for the long pulses, and $15 \mathrm{~ns}$ for the short pulse. The interpulse delays were set to $d_{1}=6100 \mathrm{~ns}, d_{3,0}=5980 \mathrm{~ns}$, and $d_{2}=3500 \mathrm{~ns}$ for ruler $2_{1}, d_{1}=4100 \mathrm{~ns}, d_{3,0}=3980 \mathrm{~ns}$, and $d_{2}=3500 \mathrm{~ns}$ for ruler $\mathbf{4}$, and $d_{1}=4100 \mathrm{~ns}, d_{3,0}=3980 \mathrm{~ns}$, and $d_{2}=5500 \mathrm{~ns}$ for ruler $2_{3}$. These choices of $d_{1}$ suppress the effect of a low-frequency artefact, which was reported for nitroxide detected RIDME. ${ }^{56}$ The longer $d_{1}$ for ruler $2_{1}$ was required to enable reproducible fitting of the RIDME background, which is less stable for data without pronounced oscillations. The mixing time was set to $T_{\text {mix }}=400 \mu \mathrm{s}$. To remove echo crossing and phase offsets, an eight-step phase cycle was used. ${ }^{63}{ }^{2} \mathrm{H}$ ESEEM averaging was performed with the stationary echo protocol. ${ }^{56,81}$

\subsection{UV/Vis spectroscopy}

Extinction measurements of light in the ultraviolet and visible range (UV/VIS) were performed on a NanoDrop One Microvolume UV Spectrophotometer (ThermoFisher). All compounds were diluted to a final concentration of $50 \mu \mathrm{M}$ in deionised $\mathrm{H}_{2} \mathrm{O}$, and a $2 \mu \mathrm{l}$ sample was loaded for each measurement (blank: deionized $\mathrm{H}_{2} \mathrm{O}$ ). The extinction measurements were performed in triplicates. The reported peak positions of the lowest energy extinction peak correspond to the local maxima of the spectra in the range above $350 \mathrm{~nm}$, and were obtained with an automated read-out script. Additional results and interpretation of the shifts of the extinction bands are given in the ESI1. $\dagger$

\subsection{Lineshape simulations}

CW EPR lineshapes were simulated using the function pepper of the EasySpin package ${ }^{84}$ in MATLAB (The MathWorks Inc., Natick, MA, USA). The spin system was based on a spin $S=1 / 2$ and a $\mathrm{Cu}(\mathrm{II})$ nucleus $I=3 / 2$ with natural isotope abundance. Axial or rhombic $g$ and $A$ tensors as well as corresponding strains were the fitting parameters. The $g / A$ strain correlation was set to -1 because, as has been found suitable in previous studies for $\mathrm{Cu}(\mathrm{II}) .{ }^{85}$ Due to the broad CW lineshapes, unresolved nitrogen hyperfine couplings were neglected. The EasySpin function esfit was used to optimize the fitting parameters using the Nelder/Mead simplex gradient. Simulations were scaled and baseline-corrected with EasySpin.

\subsection{DFT calculations}

DFT calculations were performed on the complexes $\mathrm{Cu}$-PyMTA and $\mathrm{Cu}$-TAHA in different protonation states using ORCA. ${ }^{86}$
Geometries were optimized on the restricted Kohn-Sham level with the hybrid density functional $\mathrm{B} \mathrm{LYP}^{87-89}$ in combination with D3 dispersion correction, ${ }^{90,91}$ the continuum solvation model for water and the triple zeta basis set (def2-TZVP) ${ }^{92}$ for all atoms in the final optimization step; in case of negatively charged complexes, minimal augmentation (ma-) by diffuse functions ${ }^{93}$ was added to the basis set. The RIJCOSX approximation was used to speed up the geometry optimization with auxiliary basis sets automatically constructed by $O R C A$.

The parameters of $g$ and $A$ tensors were calculated using different functionals and basis sets to test for variations throughout the DFT calculations. $g$-Tensor calculations were performed using (ma-)def2-TZVP and the hybrid density functional B3LYP in combination with the RIJCOSX approximation or using $\mathrm{PBE0}^{94}$ without and with the RIJCOSX approximation. In addition, for the $\mathrm{Cu}(\mathrm{II})$-ion a core properties basis set with three polarization functions $\mathrm{CP}(\mathrm{PPP})^{95}$ for the copper transition metal center was tested. The $A$ tensor was calculated using the PBE0 functional in combination with the EPR II or III ${ }^{96}$ basis sets for light atoms as well as the B3LYP functional in combination with the EPR II basis set for light atoms. On the $\mathrm{Cu}$ (II)-ion either the (ma)-def2-TZVPP or the CP(PPP) basis set was applied. The results of the DFT computations are shown in ESI1. $\dagger$

\subsection{Data analysis}

3.7.1. Pulsed dipolar spectroscopy data. In this contribution we used orientation-averaging protocols via either summation of RIDME and DEER traces over several detection and pump pulse positions, or by using frequency-swept excitation of the full nitroxide spectrum as described above. This step is crucial to not further complicate the data analysis by introducing additional orientation-related parameters. For the different $\mathrm{Cu}$ (II) complexes, effective $g$-values were approximated using the principal values $g_{x}$, $g_{y}$ and $g_{z}$ obtained from lineshape analysis and averaging them according to eqn (4) over the polar angles $(\theta, \phi)$. For Cu-PyMTA based rulers $\mathbf{1}_{n}$ and $2_{n}$ we used: $g_{\text {eff, } \mathrm{Cu}}=2.131$. For the [Cu-TAHA]nitroxide ruler $4: g_{\text {eff,Cu }}=2.140$ and for the $[\mathrm{Cu}-\mathrm{TAHA}]-[\mathrm{Cu}-\mathrm{TAHA}]$ ruler 3: $g_{\text {eff, } \mathrm{Cu}}=2.138$. These values differ subtly from the isotropic $g$ values of 2.127 and 2.139 due to the tilt of the local magnetic field with respect to the external field, which was neglected in our calculations. Also, as an approximation, for nitroxides $g_{\text {NO }}$ the $g$-value of the free electron $g_{\mathrm{e}}=2.0023$ was assumed. The PDS data were analyzed using four different approaches.

In the first step all experimental traces were analyzed with the DeerAnalysis ${ }^{97}$ open-source package (version 2018) whereby the standard functionality for processing dipolar evolution data was utilized (Tikhonov regularization in the time domain using the standard $K(r, t)$ kernel which assumes only dipolar coupling as given in eqn (8). Where appropriate, $g$-values for computing the dipolar coupling constants $\omega_{\mathrm{dd}}$ (eqn (3)) were adjusted.

Second, the data were analyzed using the generic and exchange resilient neural networks as implemented in DeerAnalysis2018. ${ }^{66}$

Two further custom computational approaches were developed - the coupled exchange and distance distributions 
model and the regularization in the exchange-coupling domain procedure. These are described below.

3.7.2. The coupled exchange and distance distributions model. This approximate model exploits structural peculiarities of the studied compounds, namely their stiffness and rod-like spacer shape. Such molecules are expected to possess narrow and somewhat asymmetric distance distributions between the spacer ends. End-to-end distance distributions of that kind, featuring characteristic "shark tail" shapes, were found, for example, for sets of structurally related shape-persistent biradicals carrying nitroxide moieties at both ends. ${ }^{52,53}$ Increasing spacer lengths in the series of these biradicals resulted in increased bending of the spacers and therefore broader end-toend distance distributions and a shift of the distance distribution maximum (i.e. the most probable end-to-end spacer distances) to shorter distances than the maximum possible distance. Now, if the unpaired electron is distributed over a $\pi$-system, such a spin density distribution is expected to be more extended for more stretched conformations of the spacer that facilitate $\pi$-orbital overlap. Therefore, in the coupled exchange and distance distributions model the two distributions are assumed not to be independent of each other, but rather correlated in the following way. The most extended spacers correspond to the longest spinspin distances and are expected to contribute the largest exchange coupling values. Bending of the spacers causes a reduction of end-to-end distances and simultaneous decrease of the values of the exchange coupling. We assume a simple law for both (correlated) end-to-end distance and exchange coupling distributions, i.e. a Gaussian or Lorentzian profile. In practice, both the distance distribution ${ }^{52}$ and the distribution of exchange couplings are expected to be convoluted with an additional broadening function due to other causes. In the present study, the fit quality and the overall performance of the coupled exchange and distance distributions model was found consistently better if both distance and exchange coupling distributions were taken fully symmetric.

The coupled exchange and distance distributions model was parameterized with 4 adjustable parameters: mean and standard deviation of distance and exchange-coupling distributions (see ESI1, $\dagger$ Fig. S8). Computations were performed in frequency domain using a point-dipole approximation applied to a weighted average position of the (distributed) electron spin at both ends of the molecule. Such a point-dipole approximation is permitted if the two spin density distributions have axial symmetry with coinciding symmetry axes. Spin coupling spectra obtained from experimental time-domain form-factors by Fourier transformation were used as simulation targets. Model spectra were obtained in the following way. A distance axis representing the spin-spin distance $r$ was defined and used for generating model distributions of distances $P(r)$ and exchange couplings $J(r)$ for chosen combination of $r_{\text {mean }}, \sigma_{r}$ and $J_{\text {mean }}, \sigma_{J}$ values. Since $P(r)$ and $J(r)$ are coupled, each point of the distance distribution corresponds to a particular value from the isotropic exchange coupling distribution $J(r)$ (ESI, $\dagger$ Fig. S8). For each point in $P(r)$, an ideal Pake pattern was computed based on eqn (3); each single frequency from the pattern was combined with the corresponding (isotropic) exchange coupling value from $J(r)$ thus yielding a kernel function for a particular value of $r_{\mathrm{AB}}$. Averaging over all points of the distance distribution $P(r)$ resulted in a simulated spin-spin coupling spectrum for a given $r_{\text {mean }}, \sigma_{r}$ and $J_{\text {mean }}, \sigma_{J}$ combination.

Direct fitting to experimental frequency spectra in the 4-parameter space (i.e. $r_{\text {mean }}, \sigma_{r}$ and $J_{\text {mean }}, \sigma_{J}$ ) was prone to become stuck in local minima of the error surface. Therefore, an iterative grid-search approach was adopted. In order to identify a global minimum, initial computation was always performed for a sufficiently broad range of model parameters using a rough grid for each parameter. Subsequently, parameter variation ranges were reduced while grids were made finer in order to find the best matching model for a given experimental data set. Two representative data sets in the presence and absence of exchange couplings ([Cu-PyMTA][Cu-PyMTA] $\mathbf{1}_{n}, n=3,5$ ) were subject to analysis within the coupled exchange and distance distribution model. Details of the grid-search procedure for each case are given in ESI1. $\dagger$

3.7.3. Regularization in the exchange coupling domain. This calculation procedure was developed for the determination of isotropic exchange coupling distributions of arbitrary shape at a given narrow distance distribution. Contrary to the coupled exchange and distance distribution model, both distributions in question are considered to be uncorrelated: the same exchange coupling distribution is applied to every point of the distance distribution. This is a good approximation if the distribution of dipole-dipole couplings is much narrower than the one of exchange couplings. The exchange coupling distribution is obtained as a result of the regularization procedure in the exchange coupling domain, performed with a suitable kernel similar to how distance distributions are calculated by regularization in the distance domain using the standard dipolar coupling $K(t, r)$ kernel (eqn (8)). The exchange coupling kernel $K(t, J)_{p(r)}$ was constructed in the following way. An exchange coupling axis was generated first, wherein corresponding bounds and step size were freely adjustable. The dimension of the kernel in the time domain could be chosen freely for simulations while, when processing experimental data, the number of points and the time increment were set equal to those of the experimental trace in question and were computed on the fly. The dimensions for all experimental time traces are given in ESI, $\dagger$ Table S1. Entries of the $K(t, J)_{p(r)}$ kernel corresponding to each point of the chosen $J$-axis were computed based on eqn (9), which produces a fully orientationaveraged time-domain trace for a chosen distance distribution and the current $J$-value. The complete set of all such time traces comprises a kernel:

$$
\begin{gathered}
K(t, r)=\int_{0}^{1} \cos \left(\left(3 z^{2}-1\right) \omega_{\mathrm{dd}} t\right) \mathrm{d} z . \\
K(t, J)_{p(r)}=\int_{r_{\min }}^{r_{\max }} p(r) \int_{0}^{1} \cos \left(\left[\left(3 z^{2}-1\right) \omega_{\mathrm{dd}}+J\right] t\right) \mathrm{d} z .
\end{gathered}
$$

Prior to applying this computational approach to experimental data, it was subjected to an extensive validation using 
simulated data sets. When mathematical problems are solved by kernel inversion without regularization, the rows of the kernel must be pairwise orthogonal to ensure a stable solution. The pure dipolar evolution kernel $K(t, r)$ computed with (8) does not fulfill this condition. The solution must thus be regularized. On the contrary, a hypothetical purely exchange coupling kernel $K(t, J)$ could be constructed such that the pairwise orthogonality requirement is fulfilled thus allowing for computing correct exchange coupling distributions, potentially without regularization. However, determining the character of $P(J)$ (i.e. ferromagnetic or antiferromagnetic) would be impossible in this way as the entries corresponding to ferro- and antiferromagnetic couplings of the same strength would be exactly the same according to eqn (9) for cosine being an even function. The two situations are distinguishable only in the presence of the dipolar contribution of comparable magnitude, whose sign is known. Thus the presence of dipolar coupling contributions in the $K(t J)_{p(r)}$ kernel on one hand makes the rows of the kernel less (pairwise) orthogonal resulting in less stable solutions requiring regularization. On the other hand distinguishing between the ferro- and antiferromagnetic regimes becomes possible. Results of the calibrating simulations are summarized in ESI1. $\dagger$

\section{Experimental results and qualitative discussion}

\subsection{Ruler synthesis}

Common to all rulers used in this study are spacers consisting of para-phenylene (P) and ethynylene (E) units which provide high shape persistence ${ }^{52,98}$ and are well accessible. ${ }^{99,100}$ The lengths of the rulers are adjustable, simply by the number $n$ of the repeating units PE. ${ }^{99,100}$ All of the rulers carry short branched poly(ethyleneglycol) (PEG) side chains which provide good solubility to the rulers in water and aqueous solvents as well as in polar organic solvents.

The $\mathrm{Cu}(\mathrm{II})$-nitroxide rulers were synthesized via a reaction sequence, in which the nitroxide is attached to the spacer first and then the ligand moiety (Scheme 1). ${ }^{56}$ In this way, synthesis effort needed for the variation of the metal ion chelating moiety is kept to a minimum. Important details about this reaction sequence can be found in a recent publication which describes the synthesis of the short [Cu-PyMTA]-nitroxide and [Cu-TAHA]nitroxide rulers $2_{1}$ and $4 .^{56}$ The long [Cu-PyMTA]-nitroxide ruler $2_{3}$ was assembled largely in the same way, but the linking of the nitroxide moiety and the spacer was different. For the assembly of the short $\mathrm{Cu}$-nitroxide rulers the nitroxide was attached via amide bond formation between a spacer with a terminal amino group and 2,2,5,5-tetramethyl-3-pyrrolin-1-oxyl-3-carboxylic acid (6a), the latter in form of an $\mathrm{N}$-acylpyridinium chloride $\mathbf{6 b}$ (method A). ${ }^{56}$ In case of the assembly of the long $\mathrm{Cu}$-nitroxide ruler $2_{3}$, the nitroxide was first transformed into the $N$-(4-iodophenyl)amide 7 , which was attached to the oligoPE $8_{3}$ via a Sonogashira-Hagihara coupling reaction (method B; Scheme 1). Method B was applied when the oligoPE backbone was found to be harmed under the conditions of method A. Later it was realized that this was an effect
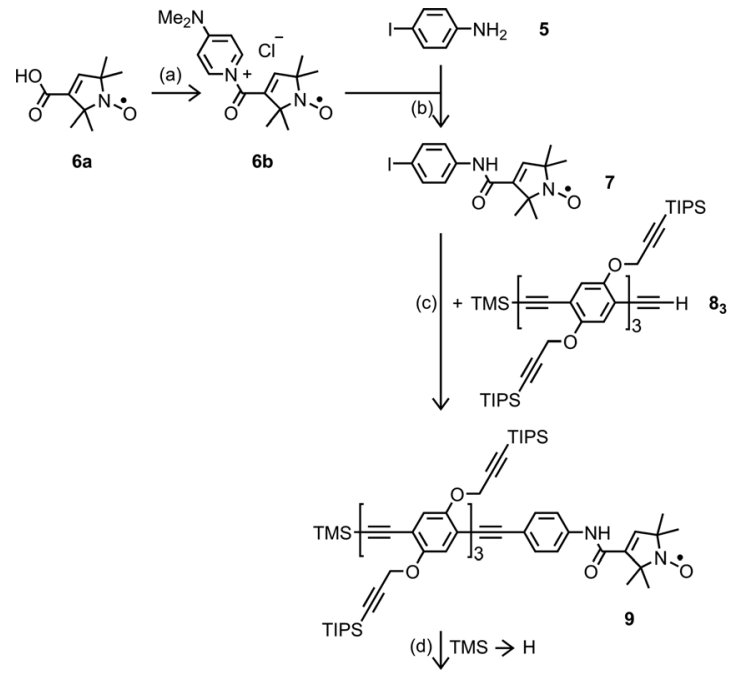

10
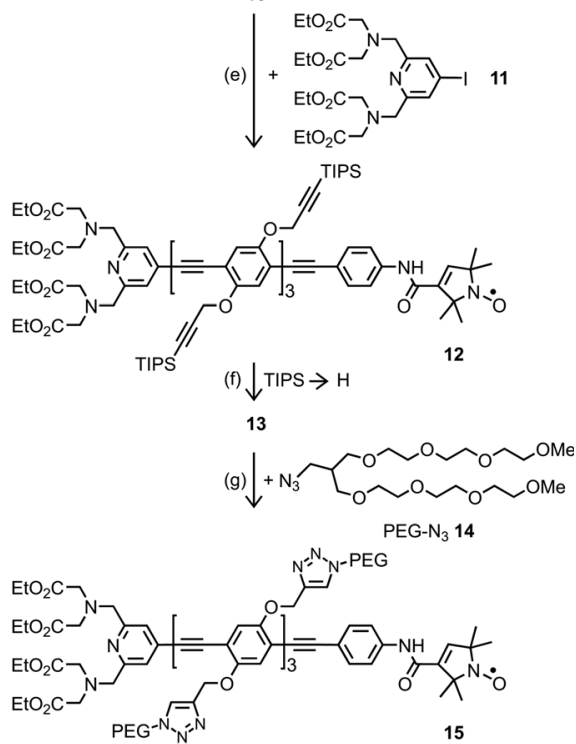

(h) $\downarrow$

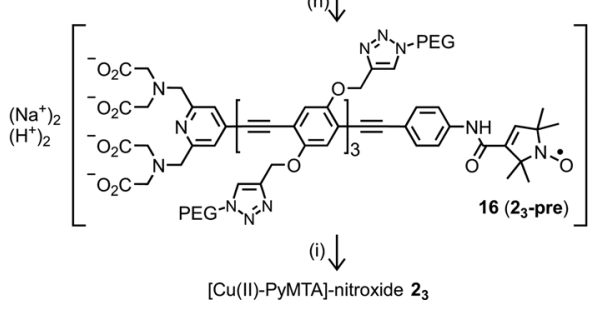

Scheme 1 Synthesis of [Cu(॥)-PyMTA]-nitroxide ruler $2_{3}$. (a) (1) DMAP, $\mathrm{CH}_{2} \mathrm{Cl}_{2}$, (2) thionyl chloride, $0{ }^{\circ} \mathrm{C}$ to $\mathrm{rt}, 65 \mathrm{~min}$; $\mathbf{6 b}$ was not isolated; (b) rt, 65 min, 98\%; (c) $\mathrm{Pd}_{2}(\mathrm{dba})_{3}, \mathrm{Cul}, \mathrm{PPh}_{3}$, piperidine, THF, rt, $45 \mathrm{~h}, 77 \%$; (d) $\mathrm{K}_{2} \mathrm{CO}_{3}, \mathrm{MeOH}, \mathrm{CH}_{2} \mathrm{Cl}_{2}, \mathrm{rt}, 14.5 \mathrm{~h}, 91 \%$; (e) (1) $\mathrm{Pd}_{2}(\mathrm{dba})_{3}, \mathrm{Cul}, \mathrm{PPh}_{3}$, ' $\mathrm{Pr}_{2} \mathrm{NH}, \mathrm{THF}, \mathrm{rt}, 45 \mathrm{~h}$, (2) 2-methylbut-3-yn-2-ol, rt, $22 \mathrm{~h}, 44 \%$; the addition of 2-methylbut-3-yn-2-ol serves to trap and thus polarly tag residual 4-iodo-PyMTA ethyl ester (11) for its easy chromatographic removal. (f) ${ }^{n} \mathrm{Bu}_{4} \mathrm{NF}$, THF, rt, $10 \mathrm{~min}$; (g) $\left[\mathrm{Cu}\left(\right.\right.$ phen) $\left.\left(\mathrm{PPh}_{3}\right)_{2}\right] \mathrm{NO}_{3} \cdot 0.5 \mathrm{CH}_{2} \mathrm{Cl}_{2}$, toluene, $67 \%$ in reference to compound 12; (h) (1) $\mathrm{NaOH}, \mathrm{EtOH}, \mathrm{H}_{2} \mathrm{O}, 18 \mathrm{~h}, \mathrm{rt}$, (2) pH adjusted to $\sim 7$ through addition of $\mathrm{HCl}$ in $\mathrm{H}_{2} \mathrm{O}, \mathrm{rt}, 80 \%$. The protonation degree at $\mathrm{pH} 7$ given with the formula of PyMTA-nitroxide 16 is based on the data for diethylenetriaminepentaacetic acid (DTPA) which is structurally similar to PyMTA. ${ }^{75}$ (i) $\mathrm{CuCl}_{2}, \mathrm{NaOD}, \mathrm{D}_{2} \mathrm{O}$, rt. For further details see $\mathrm{ESI} 2 . \dagger$ Abbrevations: $\mathrm{DMAP}=4$-(dimethylamino)pyridine, phen = phenanthroline, THF = tetrahydrofuran, TIPS = triisopropylsilyl, TMS = trimethylsilyl, $r \mathrm{t}=$ room temperature. 
of long reaction times and could be avoided by limiting the reaction time to 1-2 $\mathrm{h}$. Method $\mathrm{B}$ has its pros and cons. On the one hand it reduces the number of consecutive steps to be performed with the spacer and avoids to handle very small amounts of a highly water sensitive acylation reagent in cases where the amount of oligoPE is small. On the other hand, the Sonogashira-Hagihara coupling reaction is occasionally accompanied by reduction of a few percent of the nitroxide to hydroxylamine. ${ }^{56}$

The [Cu-PyMTA]-[Cu-PyMTA] rulers $\mathbf{1}_{n}$ were prepared in the same way as the corresponding [Gd-PyMTA]-[Gd-PyMTA] rulers, ${ }^{99}$ only using $\mathrm{CuCl}_{2}$ instead of $\mathrm{GdCl}_{3}$ in the final step. When aiming at linear rulers with two identical spin labels, as is the case for the $\mathrm{Cu}(\mathrm{II})-\mathrm{Cu}$ (II) rulers $\mathbf{1}_{n}$ and $\mathbf{3}$, an oxidative alkyne dimerization (Glaser coupling) of a building block of the type ligand-(EP) ${ }_{n} \mathrm{E}-\mathrm{H}$ which has a terminal ethyne group giving a ruler of the type ligand- $(\mathrm{EP})_{n} \mathrm{~B}(\mathrm{PE})_{n}$-ligand with $\mathrm{B}$ standing for a butadiyne unit $(\mathrm{B}=\mathrm{EE})$, is most convenient in respect to the number of synthetic steps because the spacer length is doubled in one step. The butadiyne unit makes the spacer only slightly more bendable. ${ }^{52}$ This strategy has been exploited for the synthesis of rulers with two nitroxide units. ${ }^{102}$ However, the PyMTA ester moiety turned out to be incompatible with the simultaneous presence of $\mathrm{Cu}(\mathrm{II})$ and oxygen, ${ }^{103}$ i.e. with the typical conditions for such a dimerization. Under these conditions, the aminomethyl substituent at the pyridine ring is transformed into a formyl group. ${ }^{103}$ Although being a slow side reaction, it matters because also the dimerization of long spacers proceeds rather slowly and it is difficult to remove the side product. Therefore, the short synthetic pathway was no option for the synthesis of long [Gd-PyMTA]-[Gd-PyMTA] rulers. In contrast, the TAHAester moiety proved inert under these conditions and, therefore, the oxidative dimerization was employed in the synthesis of the [Cu-TAHA]-[Cu-TAHA] ruler 3 (Scheme 2): the TAHA unit was attached to half of the final spacer via Sonogashira-Hagihara coupling of spacer building block $\mathbf{8}_{1}{ }^{99}$ with 4-iodo-TAHA ethyl ester (17) and the alkyne protecting trimethylsilyl group of coupling product 18 was removed. Glaser coupling of alkyne 19 completed the scaffold assembly. Despite the inertness of TAHA ester 17 against the simultaneous presence of $\mathrm{Cu}$ (II) salts and oxygen, we trapped all copper and palladium ions with metal scavengers during work-up of the reaction mixtures because column chromatography can be insufficient to remove the metal ions completely and even traces of residual metal ions can cause uncontrolled reactions with terminal alkyne groups. To avoid ester hydrolysis (there are six ester groups per molecule) under the basic conditions of desilylation, we applied water free potassium carbonate, thereby enabling strict exclusion of water.

After scaffold assembly, as in the synthesis of the other rulers, the triisopropylsilyl groups protecting the ethyne units in the side chains were removed and the deprotected ethyne moieties were used to attach the PEG side chains by the $\mathrm{Cu}(\mathrm{I})$ catalyzed alkyne-azide cycloaddition of alkyne 21 with PEGazide 14 giving PEGylated compound 22. The late attachment of the polar PEG side chains allowed us to use standard extraction

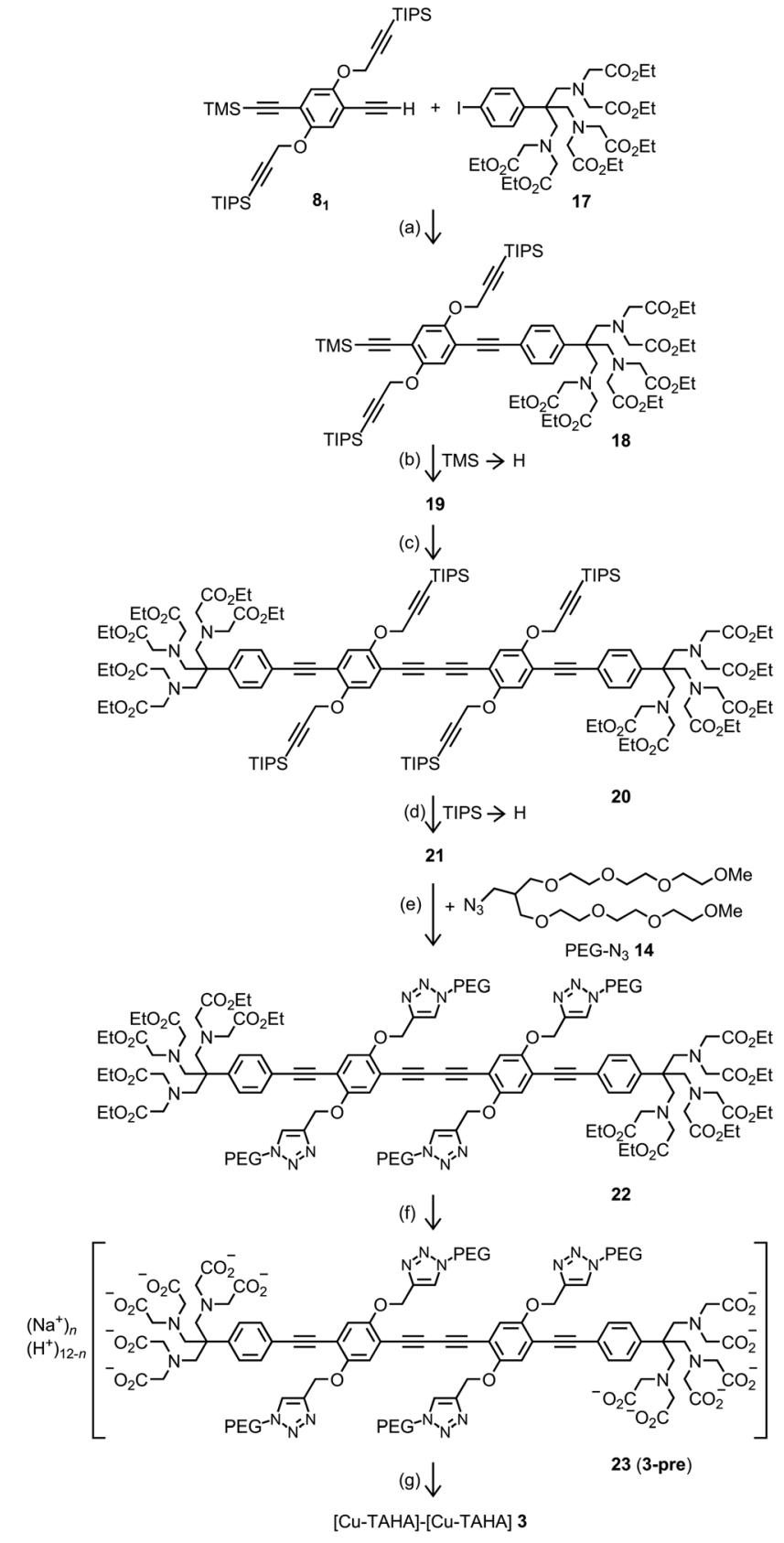

Scheme 2 Synthesis of [Cu-TAHA]-[Cu-TAHA] ruler 3. (a) $\mathrm{PdCl}_{2}\left(\mathrm{PPh}_{3}\right)_{2}$, Cul, ' $\mathrm{Pr}_{2} \mathrm{NH}$, THF, rt, 21.5 h, 82\%; (b) $\mathrm{K}_{2} \mathrm{CO}_{3}, \mathrm{EtOH}, \mathrm{THF}, \mathrm{rt}, 19 \mathrm{~h}, 95 \%$; (c) $\mathrm{PdCl}_{2}\left(\mathrm{PPh}_{3}\right)_{2}, \mathrm{Cul},{ }^{\mathrm{i}} \mathrm{Pr}_{2} \mathrm{NH}, \mathrm{THF}$, in air, rt, $23 \mathrm{~h}, 84 \%$; (d) $\mathrm{Bu}_{4} \mathrm{NF}, \mathrm{THF}, \mathrm{rt}$, $75 \mathrm{~min}$; (e) [Cu(phen) $\left.\left(\mathrm{PPh}_{3}\right)_{2}\right] \mathrm{NO}_{3} \cdot 0.5 \mathrm{CH}_{2} \mathrm{Cl}_{2}$, toluene, $30{ }^{\circ} \mathrm{C}, 69.5 \mathrm{~h}, 78 \%$ in reference to compound 20; (f) (1) $\mathrm{NaOH}, \mathrm{H}_{2} \mathrm{O}$, rt, (2) $\mathrm{HCl}$ in $\mathrm{H}_{2} \mathrm{O}$, rt, $54 \%$; the average protonation degree of TAHA-TAHA 23 is 1.7 protons per TAHA calculated with the $\mathrm{p} K_{\mathrm{a}}$ values reported for TAHA. ${ }^{101}(\mathrm{~g}) \mathrm{CuCl}_{2}, \mathrm{NaOD}, \mathrm{D}_{2} \mathrm{O}$, rt. For further details see ESI2. $\dagger$ Abbreviations: phen = phenanthroline, $\mathrm{THF}=$ tetrahydrofuran, TIPS $=$ triisopropylsilyl, $\mathrm{TMS}=$ trimethylsilyl, $\mathrm{rt}=$ room temperature.

and standard column chromatography on silica gel for purification of all products up to this step. The obtained PEGylated compound 22 was saponified to ruler precursor TAHA-TAHA 23 (denoted '3-pre' in the UV/Vis experiments) containing the free ligands ready for complexation. By addition of an aqueous 
solution of copper(II) chloride the ruler [Cu-TAHA]-[Cu-TAHA] 3 was obtained.

When preparing the rulers, about $5 \%$ less than the calculated required amount of copper salt was added to the ruler precursor in order to avoid the presence of unchelated copper ions and therefore copper ions which are spectroscopically different from the chelated ones.

\subsection{EPR lineshape and DFT calculations}

PyMTA and TAHA were used as the $\mathrm{Cu}(\mathrm{II})$ chelators. The pyridine ring of PyMTA and the benzene ring of TAHA are in conjugation with the $\pi$-system of the spacer unit. Therefore, we consider the combination of these moieties to give the effective spacer. In case of PyMTA the pyridine $\mathrm{N}$ atom can provide a path for exchange coupling if it interacts with the $\mathrm{Cu}(\mathrm{II})$ ion because it is in conjugation with the $\pi$-system of the effective spacer. Note that the amide group and the double bond in the nitroxide ring can also act as a part of the $\pi$-system, as indicated by the violet color in Fig. 1. Quite differently, all of the atoms of TAHA coordinating to the $\mathrm{Cu}(\mathrm{II})$ ion are expected to be electronically decoupled from the $\pi$-system of the spacer because of the three saturated bonds between the $\mathrm{N}$ atoms and the effective spacer.

Analysis of the $\mathrm{Cu}$ (II) EPR spectra in combination with DFT calculations was performed in order to estimate the spin density distribution at the $\mathrm{Cu}(\mathrm{II})$ complexes, and estimate the fraction of spin density transferred to the spacer's conjugated $\pi$-system. Fig. 3 shows the X-band CW EPR spectra of Cu-PyMTA (a) and Cu-TAHA (b) and their associated rulers at $140 \mathrm{~K}$. Lineshape fits are presented in ESI1. $\dagger$

The EPR spectra of Cu-PyMTA and [Cu-PyMTA]-[CuPyMTA] rulers $\mathbf{1}_{3}$ and $\mathbf{1}_{5}$ overlay well. In particular, the positions of three out of the four low-field peaks due to the $\mathrm{Cu}(\mathrm{II})$ hyperfine splitting in the parallel orientation of the $g$-frame are well resolved, and these positions are the same for $\mathrm{Cu}-\mathrm{PyMTA}$ and [Cu-PyMTA]-[Cu-PyMTA] rulers $\mathbf{1}_{3}$ and $\mathbf{1}_{5}$. The spectra of the [Cu-PyMTA]-nitroxide rulers $\boldsymbol{2}_{1}$ and $\boldsymbol{2}_{3}$ were obtained with a microwave power that heavily saturated the nitroxide radicals. Still the nitroxide line dominates the EPR lineshape around $g \approx 2$, which makes it difficult to interpret the contribution of the $\mathrm{Cu}(\mathrm{II})$ ion to this region visualized by a grey background in Fig. 3(b). However, in the parallel orientation the same three peaks due to $\mathrm{Cu}(\mathrm{II})$ hyperfine splitting are clearly visible at the same positions as for the other $\mathrm{Cu}$-PyMTA compounds. Somewhat stronger broadening is observed for these three lines of the [Cu-PyMTA]-nitroxide rulers. Importantly for our analysis, the strong hyperfine anisotropy in the Cu(II) EPR spectrum is due to the dipolar character of the hyperfine coupling, which appears due to the non-spherical distribution of the electron spin density around the $\mathrm{Cu}(\mathrm{II})$-nucleus. A strong difference in the electron spin density distribution between Cu-PyMTA and [Cu-PyMTA]-[Cu-PyMTA] ruler would result in a clear difference of this hyperfine splitting, which is not observed for the [Cu-PyMTA]-[Cu-PyMTA] rulers $\mathbf{1}_{3}$ and $\mathbf{1}_{5}$. Thus, also in case of these rulers the major part of the electron spin density must be limited to the atoms of the Cu-PyMTA moiety. On the other hand, the lineshape of [Cu-PyMTA]-[Cu-PyMTA] ruler $\mathbf{1}_{1}$, which has the shortest $\mathrm{Cu}-\mathrm{Cu}$ distance, strongly deviates from the one of the [Cu-PyMTA]-[Cu-PyMTA] rulers $\mathbf{1}_{3}$ and $\mathbf{1}_{5}$ featuring longer spacers. This indicates that the two $\mathrm{Cu}(\mathrm{II})$ centres in this compound are significantly exchange-coupled. The broadening due to pure dipole-dipole interaction at the anticipated $\mathrm{Cu}$ (II)-Cu(II) mean distance of about $2.1 \mathrm{~nm}$ would not be sufficient to change the CW EPR lineshape of ruler $\mathbf{1}_{1}$ that strong. Spin-density transfer into the ruler $\pi$-system is an explanation of the EPR lineshape change for the ruler $\mathbf{1}_{1}$. Also, a large electron-electron coupling is consistent with a strongly reduced modulation depth in PDS experiments (see ESI + ), as large couplings cannot be excited by the applied microwave pulses. ${ }^{104}$ Calculation of a broad unknown distance distribution along with simultaneous determination of a distribution of exchange couplings would be highly ambiguous, since any distribution of dipolar couplings can be substituted by an appropriate distribution of exchange couplings. The DFT-based estimates of the spin density distributions in such samples would not provide sufficient accuracy of the dipole-dipole coupling estimate to stabilize the fitting procedure. Even more importantly, a set of thermally excitable geometries would need to be considered in order to obtain an (a)

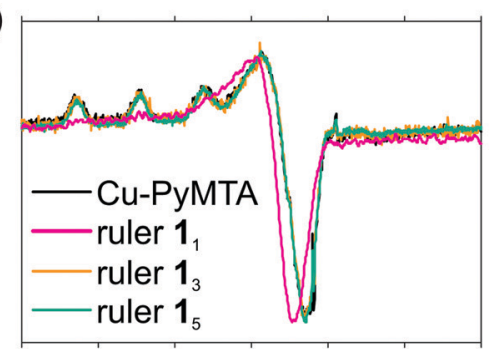

260280300320340360380 (b)

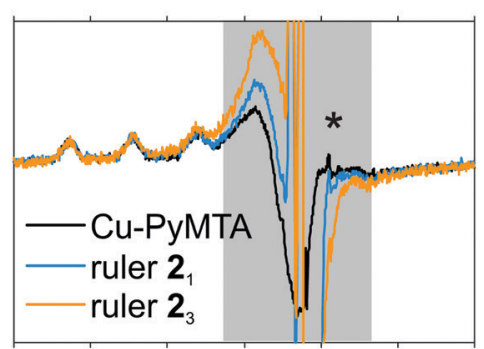

260280300320340360380 (c)

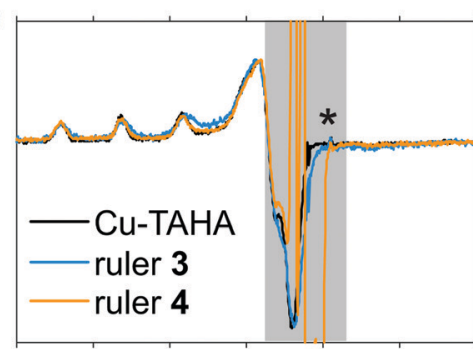

260280300320340360380

\section{$\mathrm{B} / \mathrm{mT}$}

Fig. 3 Low temperature (140 K) CW EPR spectra of $\mathrm{Cu}(॥)-\mathrm{Cu}(॥)$ rulers and the corresponding $\mathrm{Cu}(॥)$ complexes in X band. (a) Cu-PyMTA (black) and the [Cu-PyMTA]-[Cu-PyMTA] rulers: $\mathbf{1}_{1}$ (magenta), $\mathbf{1}_{3}$ (orange) and $\mathbf{1}_{5}$ (green); (b) Cu-PyMTA (black) and the [Cu-PyMTA]-nitroxide rulers: $\mathbf{2}_{1}$ (blue) and $\mathbf{2}_{3}$ (orange); (c) $\mathrm{Cu}-\mathrm{TAHA}$ (black), [Cu-TAHA]-[Cu-TAHA] ruler $\mathbf{3}$ (blue) and [Cu-TAHA]-nitroxide ruler $\mathbf{4}$ (orange). The grey shading marks the area of intense overlap with the nitroxide spectrum in the $\mathrm{Cu}$-nitroxide rulers; the asterisk marks a background artefact. 
estimate of the distribution of dipole-dipole couplings. We thus refrained from detailed analysis of [Cu-PyMTA]-[Cu-PyMTA] ruler $\mathbf{1}_{1}$ by PDS experiments.

The Cu-PyMTA spectrum was analysed previously ${ }^{33}$ and is best reproduced using a rhombic $g$ tensor $\left(g_{x}=2.048, g_{y}=2.078\right.$, $\left.g_{z}=2.253\right)$ as well as a rhombic $A$ tensor $\left(A_{x} \sim 25 \mathrm{MHz}, A_{y} \sim\right.$ $\left.75 \mathrm{MHz}, A_{z} \sim 510 \mathrm{MHz}\right)$. Note however that the broad lines render extraction of the perpendicular tensor components ambiguous (see $\mathrm{ESI} \dagger$ for details).

Concerning the TAHA-containing compounds, the EPR spectrum for the [Cu(II)-TAHA]-nitroxide ruler 4 does almost perfectly overlay with the corresponding EPR spectrum of $\mathrm{Cu}$-TAHA if the contribution of the nitroxide is neglected. The lineshape simulation for Cu-TAHA gives axial $g$ and $A$ tensors ( $\left.g_{x, y}=2.066, g_{z}=2.284, A_{x, y}=23 \mathrm{MHz}, A_{z}=498 \mathrm{MHz}\right)$. In the case of [Cu-TAHA]-[Cu-TAHA] ruler 3 some broadening and a slight shift of the parallel Cu-hyperfine components occur relative to the other two TAHA-based compounds. Thus, for simulating the spectrum of [Cu-TAHA]-[Cu-TAHA] ruler 3 it is not sufficient to only adjust strains, but a small shift in $g_{z}$ and $A_{z}$ is needed (see ESI $1 \dagger$ ). Nevertheless, since this shift is small we can assume that the spin density transfer into the effective spacer must be weak in the series of [Cu-TAHA]-based compounds.

The clearly visible components of the $\mathrm{Cu}(\mathrm{II})$ hyperfine quartet in the parallel region with peak positions determined by $g_{z}$ and $A_{z}$ serve as a fingerprint for the local $\mathrm{Cu}$ (II) coordination according to the analysis by Peisach and Blumberg. ${ }^{105}$ Analyzing $g_{z}$ and $A_{z}$ of $\mathrm{Cu}$-PyMTA and Cu-TAHA accordingly shows (ESI1, $\dagger$ Fig. S24) that both complexes fall into an ambiguous region with three or two directly coordinating nitrogens, with a tendency more towards three coordinating nitrogen atoms for $\mathrm{Cu}-\mathrm{PyMTA}$ and more towards two coordinating nitrogen atoms for $\mathrm{Cu}$-TAHA.

The degree of spin delocalization was also studied by DFT calculations of $\mathrm{Cu}$-PyMTA and $\mathrm{Cu}$-TAHA. $\mathrm{Cu}-\mathrm{PyMTA}$ is assumed to be fully deprotonated at $\mathrm{pH} 7$ because a complete deprotonation is reported for the structurally similar complex Cu(II)-DTPA with DTPA being diethylenetriaminepentaacetic acid. ${ }^{75}$ For $\mathrm{Cu}$-TAHA on average 1.7 protons are anticipated. ${ }^{56}$ Nevertheless, in solution an equilibrium of different protonation states is expected and thus geometries for the complexes with different numbers of protons were considered. The optimized geometries were sensitive to the protonation degree, which also led to differences in the tensor symmetries and calculated values of $g, A_{\mathrm{N}}$ as well as $A_{\mathrm{Cu}}$ (see ESI $1 \dagger$ ). The $g$ and $A_{\mathrm{N}}$ parameters are relatively stable for different functionals and basis sets, but $A_{\mathrm{Cu}}$ varies strongly and in some cases is qualitatively wrong for the TZVPP basis, indicating that the degrees of freedom in this basis, particularly in the core region, are insufficiently accurate to model core properties such as metal hyperfine couplings. For some of the protonation states, the calculated $g$ and $A_{\mathrm{Cu}}$ tensor symmetries match the experimental data qualitatively. However, we did not find sufficient quantitative agreement to differentiate between different structures. For $\mathrm{Cu}-\mathrm{PyMTA}$ and [Cu-TAHA]-[Cu-TAHA] ruler 3, adding the largest DFT calculated ${ }^{14} \mathrm{~N}$ hyperfine values $(\sim 50 \mathrm{MHz})$ to the lineshape simulation is in agreement with the experimental data, i.e. this ${ }^{14} \mathrm{~N}$ hyperfine coupling can be accommodated as an unresolved coupling in the lineshape fits. Similarly, for $\mathrm{Cu}$-TAHA an experimental upper limit of about $40 \mathrm{MHz}$ is found for the $z$-component of the nitrogen hyperfine coupling or an $A_{\text {iso }}$ of $35 \mathrm{MHz}$ (in DFT the largest ${ }^{14} \mathrm{~N}$ hyperfine coupling is $\sim 44 \mathrm{MHz}$, see ESI $1 \dagger$ ). This suggests that no spin density on nitrogen atoms beyond $13 \%$ for $\mathrm{Cu}$-PyMTA and [Cu-TAHA][Cu-TAHA] ruler 3 and beyond $10 \%$ for $\mathrm{Cu}-\mathrm{TAHA}$ is expected. This is in agreement with the Mulliken population analysis that shows throughout all studied cases a spin density of $60-80 \%$ on the $\mathrm{Cu}(\mathrm{II})$-ion and no significant spin density on atoms beyond the first coordination sphere. Spin density distributions are shown in ESI1, $\dagger$ Fig. S25 and S26. This indicates that the spin density remains approximately within a sphere with a radius of about 2-3 $\AA$ around the $\mathrm{Cu}(\mathrm{II})$ ion and strongly peaks at the ion. Compared to a spin-spin distance of $2.4 \mathrm{~nm}$ or larger such an extension in space is rather small. Furthermore, this distribution is within the most narrow distance distribution widths computed from the PDS data of these rulers (see the following sections). Thus, taken together with the results of the CW EPR lineshape analysis, a point-dipole approximation with localization of the spin density at the copper ions appears to be a good approximation and will be assumed in the following analysis of PDS data for this series of compounds.

Note that $\mathrm{Cu}$ (II) coordination can be fairly variable in terms of changes in bond lengths and bond angles. This can lead to a distribution in $g$-tensor value and, more importantly, of orientations. ${ }^{106}$ Here we strongly reduce the effect of orientations by the RIDME-based effective broad-band measurements and by the detection position averaging. Additionally, the well resolved spectral features in the $\mathrm{Cu}$ (II) EPR spectra also indicate rather narrow $g$-values distributions, which in turn suggest rather low complex plasticity. Furthermore, we did not observe any measurable contribution of the free Cu(II) EPR signal in the CW EPR spectra of studied model compounds. This suggests rather low $K_{\mathrm{d}}$ for the $\mathrm{Cu}(\mathrm{II})$ chelates studied: $K_{\mathrm{d}}$ here cannot be higher than 1-2 $\mu \mathrm{M}$, and more realistically it lies yet lower, somewhere in the $\mathrm{nM}$ range.

\subsection{UV/Vis spectroscopy data}

The visual inspection of aqueous solutions of the ruler precursors, i.e. the compounds before loading them with $\mathrm{Cu}$ (II) ions to obtain the rulers (colorless for precursor 4-pre of ruler 4, very pale yellow for precursor $2_{1}$-pre of ruler $2_{1}$, pale yellow for the precursors $\mathbf{1}_{3}$-pre and $\mathbf{2}_{3}$-pre of rulers $\mathbf{1}_{3}$ and $\mathbf{2}_{3}$, and intense yellow for the precursor $\mathbf{1}_{5}$-pre of ruler $\mathbf{1}_{5}$ ) suggested that the light extinction properties of the rulers in the visible range are dominated by the electrons in the conjugated $\pi$ system of the effective spacer. This led us to perform UV/Vis measurements to determine the changes in the light extinction properties upon complexation of $\mathrm{Cu}$ (II) ions by the chelators PyMTA and TAHA of the ruler precursors giving the corresponding rulers, which may report on the weak interactions of the conjugated $\pi$ system with the $\mathrm{Cu}$ (II) ions. However, we point out that effects can be subtle or hidden in the UV/Vis spectra, because we observe 
excitation of the entire conjugated $\pi$-system, not only the unpaired electron, which is relevant for EPR. Second, the UV/Vis spectra depend not only on the electronic configuration of the ground state, but also on the electronic configuration of the excited state, where stronger delocalization of the unpaired electron of the $\mathrm{Cu}$ (II) ion cannot be excluded a priori.

The UV/Vis spectra of the rulers and the ruler precursors are shown in Fig. 4. As predicted, the long wavelength cutoff in the extinction spectra (which corresponds to the lowest energy excitation) correlates with the number of spacer units $n$ for each ruler type: the longer the ruler, the longer the cutoff wavelength. This is in agreement with UV/Vis studies of conjugated polymers. ${ }^{107-109}$ A list of the lowest energy peak wavelengths can be found in ESI1. $\dagger$ Note that the near UV spectral range ( $\lambda=300 \mathrm{~nm}$ and below) is shown, but not analyzed in the following, and the spectra in this range do not vary significantly between different rulers.

In the case of the TAHA-based compounds (ruler precursors 3-pre and 4-pre and the corresponding rulers 3 and 4) loading of the ruler precursors with $\mathrm{Cu}(\mathrm{II})$ does not affect the longwavelength cutoff area of the UV/Vis spectra significantly. In the case of PyMTA-based compounds (ruler precursors $\mathbf{1}_{n}$-pre and $\mathbf{2}_{n}$-pre with $n=1,3,5$, and the corresponding rulers $\mathbf{1}_{n}$ and $\mathbf{2}_{n}$ ) we observed weak red-shifts of the long wavelength cutoff upon loading the ruler precursors with $\mathrm{Cu}(\mathrm{II})$. This red-shift is larger for the ruler precursor/ruler pairs with shorter spacer lengths. The largest absolute shifts are observed for the pairs $\mathbf{1}_{1}$-pre/ $\mathbf{1}_{1}$ (Fig. 4(c), red-shift of $6.8 \mathrm{~nm}$ ) and $2_{1}$-pre/2 $\mathbf{2}_{1}$ (Fig. 4(c), red-shift of $4.3 \mathrm{~nm}$ ). For the ruler precursor/ruler pairs with intermediate spacer lengths the red-shift is smaller, but still clearly visible (ruler precursors $\mathbf{1}_{3}$-pre and $\mathbf{2}_{3}$-pre and the corresponding rulers $\mathbf{1}_{3}$ and $2_{3}$, Fig. $4(\mathrm{~b}$ and c)). The red-shift of the long wavelength cutoff for the pair ruler precursor $\mathbf{1}_{3}$-pre/ruler $\mathbf{1}_{3}$ with two PyMTA moieties, one at each end of the spacer, is slightly larger than the corresponding red-shift for the case of the pair ruler precursor $2_{3}$-pre/ruler $2_{3}$ with only one PyMTA moiety and one nitroxide moiety at the other end of the spacer. Note that loading of the ruler precursor corresponds to the addition of two $\mathrm{Cu}(\mathrm{II})$ metal ions in the first case, and of only one metal ion in the latter case. For the pair with the longest spacer, ruler precursor $\mathbf{1}_{5}$-pre/ruler $\mathbf{1}_{5}$, the red-shift is nearly invisible.

The maximum strength of the spin-spin couplings for the studied rulers is below $20 \mathrm{MHz}$ (see the following sections). This indicates that the electron spin density of the $\mathrm{Cu}(\mathrm{II})$ ion is only weakly coupled to the $\pi$-system of the effective spacer in the electronic ground state. However, first, in the UV/Vis spectra we observe excitation of the entire conjugated $\pi$-system, not only the unpaired electron, which is relevant for EPR. Second, the UV/Vis spectra depend not only on the electronic configuration of the ground state, but also on the electronic configuration of the excited state, where stronger delocalization of the unpaired electron of the $\mathrm{Cu}$ (II) ion cannot be excluded a priori. Thus, the overall picture given by the UV/Vis data is in line with the assumption of the weak exchange between paramagnetic centres in these compounds.

\subsection{Pulsed dipolar spectroscopy data}

Fig. 5 shows the PDS data for the studied compounds. The rulers [Cu-TAHA]-nitroxide 4, [Cu-PyMTA]-nitroxide $2_{3}$ and [Cu-PyMTA][Cu-PyMTA] $\mathbf{1}_{5}$ exhibit long-lasting oscillations, which result in narrow, single-peak distance distributions. On the other hand, [Cu-TAHA]-[Cu-TAHA] ruler 3, [Cu-PyMTA]-nitroxide ruler $\mathbf{2}_{1}$ and [Cu-PyMTA]-[Cu-PyMTA] ruler $\mathbf{1}_{3}$ show damped oscillations indicating that a distribution of spin-spin couplings is present. Furthermore, corresponding distance calculations using a dipolar coupling kernel $\left(K(t, r)=\int_{0}^{1} \cos \left(\left(3 z^{2}-1\right) \omega_{\mathrm{dd}}(r) t\right) \mathrm{d} z\right)$, as, for example, implemented in DeerAnalysis, ${ }^{97}$ feature broad distance distributions with multiple (potentially artefact) peaks.

Given the identical architecture of the PyMTA-based compounds with different spacer lengths, it is excluded that the shorter spacers exhibit higher flexibility, and the corresponding compounds possess truly broader distributions of spin-spin distances to which such a faster dampening could be related. Indeed, the measured distance distribution widths should be (a) TAHA-TAHA/nitroxide

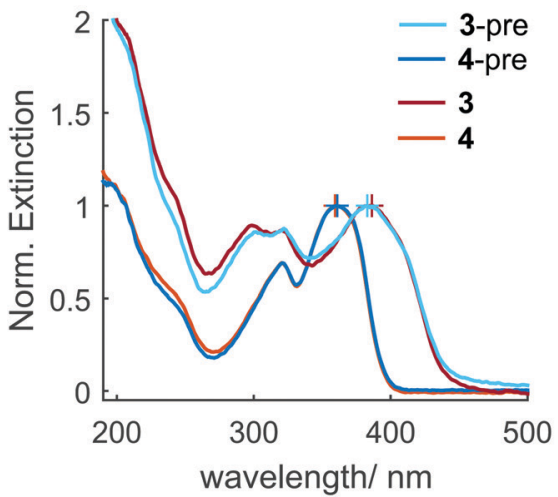

(b) PyMTA-nitroxide

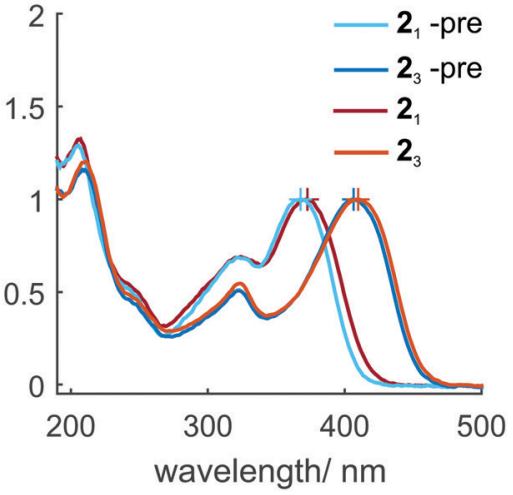

(c) PyMTA-PyMTA

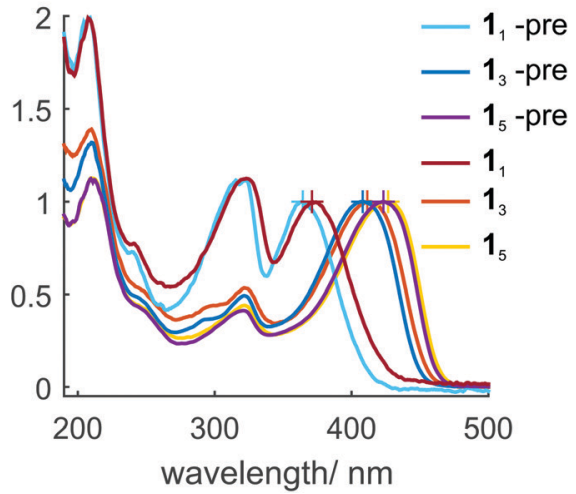

Fig. 4 UV/Vis spectra of the ruler precursors ('-pre') and corresponding rulers, normalized to the maximum of the lowest energy peak of each individual trace (indicated by cross). Ruler precursors and rulers (a) with TAHA ligands; (b) with one PyMTA ligand and one nitroxide moiety; (c) with two PyMTA ligands; all maxima and shifts are tabulated in ESI1.† 
(a)

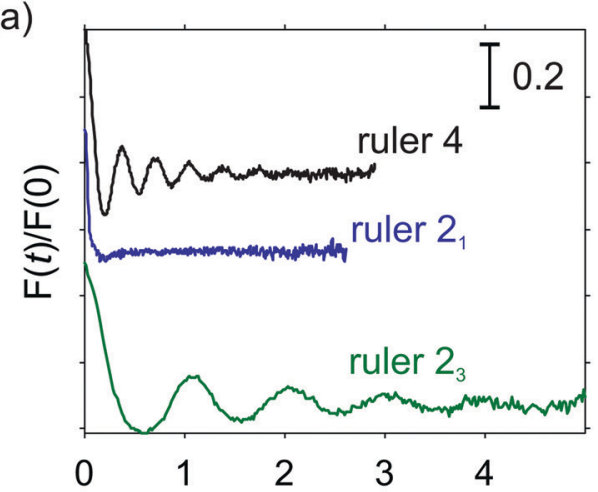

(c)

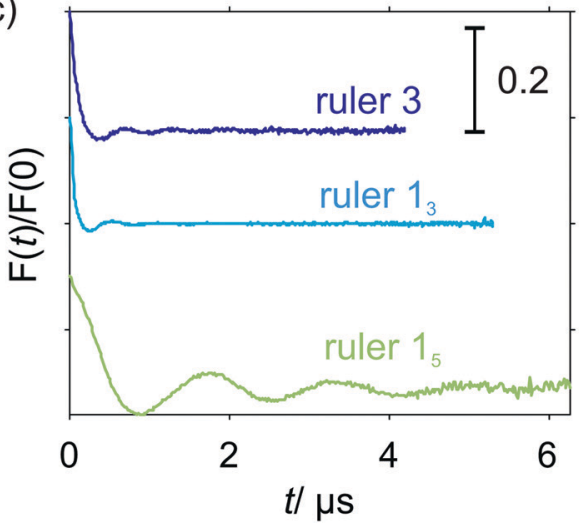

(b)

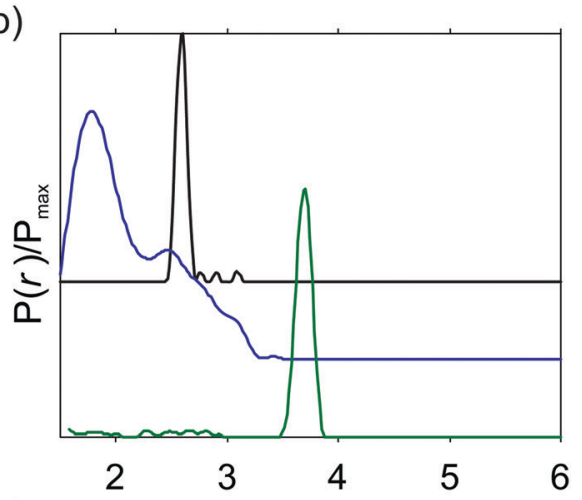

(d)

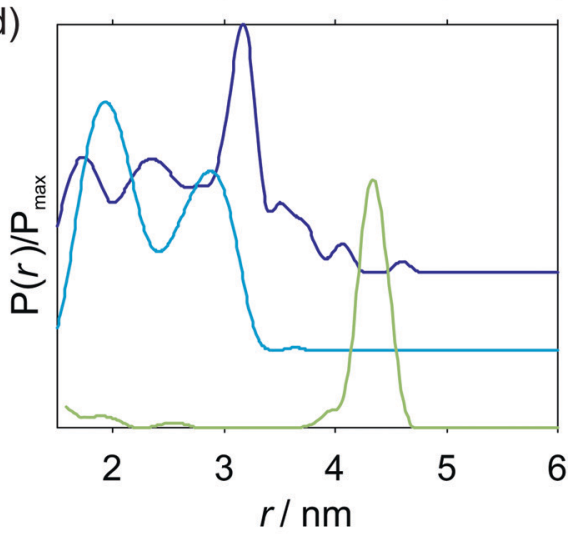

Fig. 5 PDS data for the studied ruler compounds (see ESI $1 \uparrow$ for the primary data). (a and c) Background-corrected RIDME form factors F(t) and (b and d) apparent distance distributions $P(r)$ computed neglecting exchange coupling. (a and b) Molecular rulers with one paramagnetic center being Cu(॥) and one being nitroxide: [Cu-PyMTA]-nitroxide rulers $\mathbf{2}_{n}(n=1,3)$ and [Cu-TAHA]-nitroxide ruler $\mathbf{4}$. (c and d) Both paramagnetic centers are $\mathrm{Cu}(\mathrm{II})$ : $[\mathrm{Cu}-\mathrm{PyMTA}]-[\mathrm{Cu}-\mathrm{PyMTA}]$ rulers $\mathbf{1}_{n}(n=3,5),[\mathrm{Cu}-\mathrm{TAHA}]-[\mathrm{Cu}-\mathrm{TAHA}]$ ruler 3.

comparable to those found for a series of stiff rod-like nitroxide biradicals exhibiting pure dipole-dipole character of spin-spin couplings..$^{52}$ Neither is a higher flexibility of these spacers supported by earlier distance measurements on the same [M-PyMTA]-[M-PyMTA] compounds using $\mathrm{M}=\mathrm{Gd}(\mathrm{III})^{71,110}$ or $\mathrm{Mn}(\mathrm{II})^{81}$ as metal centers. These measurements resulted in metal-to-metal distance distributions of similar widths centered around $3.4 \mathrm{~nm}$ and $4.7 \mathrm{~nm}$ for the short and long spacer, respectively. In contrast, it was shown earlier ${ }^{37}$ that such a dampening can result from distributed J-couplings. In conjugated $\pi$-systems - as present in the [Cu-PyMTA]-nitroxide and the [Cu-PyMTA]-[Cu-PyMTA] rulers - non-negligible contributions from exchange couplings have been observed for spinspin distances up to $3.6 \mathrm{~nm} .{ }^{45}$ The exponential decay of the exchange coupling with distance or a number of structurally identical linker units would be able to explain its absence for the compounds with longer spacers. ${ }^{44,51}$

Very similar qualitative conclusions can be made for the present series of compounds simply based on the apparent distance distributions presented in Fig. 5, and on the nearly identical CW EPR spectra within the series of PyMTA-based and TAHA-based compounds. First, the Cu(II) CW EPR spectra of the PyMTA-based rulers $\mathbf{2}_{1}, \mathbf{2}_{3}, \mathbf{1}_{3}$ and $\mathbf{1}_{5}$ are essentially the same, which strongly suggests that all $\mathrm{Cu}-\mathrm{PyMTA}$ containing rulers show the same distribution of conformations of the $\mathrm{Cu}-\mathrm{PyMTA}$ moiety, and the same holds true for all $\mathrm{Cu}$-TAHA containing rulers. Since the PyMTA-based rulers exhibit narrow apparent distance distributions when the spacer is long and broad ones when it is short, it is reasonable to assume that the apparent short spin-spin distances in the latter case result from distortion of the dipolar spectra by exchange interaction, whereas the true distribution of spin-spin distances is narrow. Second, the apparent distance distributions show either distances that are close to the expected spin-spin distance or shorter. However, the spin-spin distances which are predicted based on the compound structures and which are experimentally observed in the case of the longer spacers, correspond to the nearly closest placement of the $\mathrm{Cu}(\mathrm{II})$ ion with respect to the partner $\mathrm{Cu}(\mathrm{II})$ or nitroxide spin. Therefore, yet significantly shorter spin-spin distances are not feasible with the given structures of the ruler compounds. Since apparent short distances in the distance distributions in Fig. 5 correspond to stronger spinspin couplings than expected from dipolar interaction, we conclude that exchange interaction is the reason for the broad apparent distance distributions.

If we interpret the widths of the apparent distance distributions in Fig. 5 as a measure of the strengths of exchange couplings, some further useful comparisons can be made. In the case of [Cu-TAHA]-nitroxide ruler 4 and [Cu-TAHA][Cu-TAHA $]$ ruler 3 , the three single bonds between the $\mathrm{N}$ atoms 
being in contact with the $\mathrm{Cu}(\mathrm{II})$ ion and the effective spacer are expected to decouple the conjugated $\pi$-system of the spacer from the unpaired electron of the $\mathrm{Cu}$ (II) ion. This is supported by the UV/Vis data described above that do not show a red-shift in the absorption spectra for the rulers $\mathbf{3 / 4}$ involving at least one TAHA ligand as compared to corresponding ruler precursors. Thus, the range of spacer lengths for which we expect exchange coupling is presumed to be much shorter. However, while UV/Vis spectra can reveal conjugation of the $\mathrm{Cu}$ (II) ion with the $\pi$-system of the effective spacer, as found for the rulers with $\mathrm{Cu}$ (II)-PyMTA moieties, they would not be able to detect weak exchange coupling through space or through saturated bonds, which likely takes place for the rulers with $\mathrm{Cu}$-TAHA moieties. The assumption of weak exchange coupling when $\mathrm{Cu}$-TAHA is present is supported by the observation of a narrow single peak in the apparent distance distribution of [Cu-TAHA]-nitroxide ruler 4 . In contrast, [Cu-TAHA]-[Cu-TAHA] ruler 3 shows several additional peaks contributing to a broad background next to a relatively narrow peak at $3.3 \mathrm{~nm}$, the latter being in agreement with the distance anticipated based on the molecular structure. The comparison of the PDS data for the two $\mathrm{Cu}(\mathrm{II})$-nitroxide rulers $2_{1}$ and 4 allows us to conclude that coupling of the $\mathrm{Cu}$ (II)-TAHA spin with the spacer $\pi$-system is weaker than for $\mathrm{Cu}$ (II)-PyMTA. The same conclusion can be made by comparing the PDS data of [Cu-PyMTA]-[Cu-PyMTA] ruler $\mathbf{1}_{3}$ and $[\mathrm{Cu}-\mathrm{TAHA}]-[\mathrm{Cu}-\mathrm{TAHA}]$ ruler 3 . The broad distance peak obtained for [Cu-TAHA]-[Cu-TAHA] ruler 3 overlaps with the more narrow peak at the anticipated spin-spin distance, while for [Cu-PyMTA]-[Cu-PyMTA] ruler $\mathbf{1}_{3}$ the anticipated distance peak is not well visible, rather a broad exchange-distorted distribution is observed. This indicates that the mean exchange coupling and the width of the exchange coupling distribution is smaller for $\mathbf{3}$ as compared to $\mathbf{1}_{3}$ in agreement with the interruption of the conjugation between the $\pi$-system of the effective spacer and the $\mathrm{Cu}(\mathrm{II})$ ion in the $\mathrm{Cu}$-TAHA moiety.

Furthermore, based on stronger distortions of the apparent distance distribution, we can state that the exchange interaction between two $\mathrm{Cu}$-TAHA complexes (ruler 3) is stronger than between one $\mathrm{Cu}$-TAHA complex and one nitroxide moiety (ruler 4). Note that the expected spin-spin distance in [Cu-TAHA $]-[\mathrm{Cu}-\mathrm{TAHA}]$ ruler $\mathbf{3}$ is even longer than in the [Cu-TAHA]-nitroxide ruler $\mathbf{4}$, which makes the last conclusion even stronger. The comparison of [Cu-PyMTA]-[Cu-PyMTA] with [Cu-TAHA]-[Cu-TAHA] is also in line with stronger coupling to the spacer conjugated $\pi$-system for Cu-PyMTA moieties as compared to the Cu-TAHA moieties. To summarize these observations, the Cu-TAHA complex exhibits a weaker exchange coupling with the nitroxide moiety than the Cu-PyMTA complex and the exchange coupling is weaker in $\mathrm{Cu}(\mathrm{II})$-nitroxide than in $\mathrm{Cu}$ (II)-Cu(II) pairs. Thus, the propensity to induce exchange interaction with the partner moiety in the studied series of three paramagnetic units has the order nitroxide $<$ $\mathrm{Cu}$-TAHA $<\mathrm{Cu}$-PyMTA.

Possible distortions of the PDS data can be excluded as follows. Note that apart from the contribution of spectral diffusion to the RIDME experiment, which results in a faster, strongly curved background shape, orientation-averaged RIDME data are semi-quantitatively concurrent with orientation-averaged DEER data of all measurements in this work (see ESI1, $\dagger$ Fig. S1 and S2). This strongly indicates that the additional peaks in some of the cases result neither from incomplete ESEEM averaging nor from orientation selection effects. These conclusions are further supported by RIDME background reference measurements for $\mathrm{Cu}$-TAHA as well as $\mathrm{Cu}$-PyMTA shown in ESI1, $\dagger$ Fig. S7. Aggregation of the rulers appears unlikely considering the solvent polarity. Moreover, the nitroxide-nitroxide DEER measurements with the $\mathrm{Cu}(\mathrm{II})$-nitroxide rulers $2_{1}$ and 4 did not reveal any dipolar modulation above the noise level as presented in ESI1. $\dagger$ Note further that for the $\mathrm{Cu}(\mathrm{II})-\mathrm{Cu}(\mathrm{II})$ and $\mathrm{Cu}(\mathrm{II})$-nitroxide rulers $\mathbf{1}_{5}, \mathbf{2}_{3}$ and $\mathbf{4}$ we see pure dipolar couplings corresponding to the anticipated narrow spin-spin distance distributions and hence no indications for aggregation.

We cannot fully exclude some weak residual contributions from orientation selection. However, such a contribution cannot explain the observed broad apparent distance distributions (see additional analysis in ESI $1 \dagger$ ). Finally, we considered whether the partially charged end groups in the TAHA moiety might influence spacer conformation and thus cause additional peaks in the distance distribution. With a Bjerrum length of $7.1 \AA$ in water and a dielectric constant of about 60 of the water/glycerol mixtures ${ }^{111}$ that we use, one expects that the Coulomb interaction between two unit charges drops below thermal energy at about $9 \AA$. Since the distances between copper ions are much larger in all ruler compounds, we disregard such electrostatic effects.

In the following we report on the performance of our data analysis routines to extract exchange couplings, assuming that all mentioned distortions are weak and do not change the overall picture.

\section{Quantitative analysis of exchange couplings}

Straightforward processing of the time-domain PDS data of [Cu-PyMTA]-[Cu-PyMTA] compounds $\mathbf{1}_{n}$ using a standard dipolar coupling kernel and Tikhonov regularization in the distance domain as shown in Fig. 5 gives a single narrow peak at around $4.5 \mathrm{~nm}$ for the compound with the longest spacer $\mathbf{1}_{5}$. The mean metal-metal distance shortening of about $2 \AA$ for this $\mathrm{Cu}(\mathrm{II})$ loaded compound ( $1 \AA$ per moiety) as compared to the corresponding $\mathrm{Mn}$ (II)- and Gd(III)-loaded compounds $(\sim 4.7 \mathrm{~nm})$ is larger than expected based on the smaller ion radius of $\mathrm{Cu}$ (II) $(0.71 \AA$ vs. $0.80 \AA$ for $\mathrm{Mn}(\mathrm{II})$ and $1.19 \AA$ for $\mathrm{Gd}(\mathrm{III})$ ) and the higher affinity of $\mathrm{Cu}$ (II) to nitrogen atoms, which might move the $\mathrm{Cu}$ (II) ion closer to the pyridine ring. In fact, one would expect a stronger shift from these contributions between $\mathrm{Gd}(\mathrm{III})$ and $\mathrm{Mn}$ (II) than between $\mathrm{Mn}$ (II) and $\mathrm{Cu}$ (II). Hence, the shorter apparent distance is likely a result of higher spin density at the nitrogen atoms of the PyMTA ligand. However, the DFT calculations in combination with lineshape simulations described above gave an estimate of a maximum spin density at the nitrogen atoms of about $13 \%$. 
With the length of a dative bond of only $2 \AA$ we cannot exclude that part of the experimentally observed shift stems from delocalization of spin density into the effective spacer. Note further that $g$-values were not adjusted in the analysis of the PDS data for Mn(II)- and Gd(III)-based compounds.

The apparent distance distribution of the shorter compound $\mathbf{1}_{3}$ is broad and bimodal. The longer-distance peak, centered at about $3 \mathrm{~nm}$, roughly agrees with the anticipated distance for this ruler, but it is too broad, while the second peak is also broad and shifted towards shorter distances. This is taken as indication of the presence of distributed exchange couplings which may, in combination with dipolar couplings, result in the broadly distributed total spin-spin coupling frequencies. Such frequencies may or may not coincide with dipolar couplings expected for the ruler $\mathbf{1}_{3}$ in the absence of exchange coupling. One may further speculate that the presence of the distance band at around $3 \mathrm{~nm}$ in this case may correspond to a fraction of rulers with weaker exchange couplings distributed around zero. Based on this preliminary inspection of corresponding experimental data, [Cu-PyMTA]-[Cu-PyMTA] compounds $\mathbf{1}_{5}$ and $\mathbf{1}_{3}$ could serve as test cases for situations of the absence of exchange coupling (for $\mathbf{1}_{5}$ ) and the presence of distributed exchange couplings (for $\mathbf{1}_{3}$ ).

\subsection{Model of coupled exchange and distance distributions}

Spin coupling spectra of [Cu-PyMTA]-[Cu-PyMTA] compounds $\mathbf{1}_{3}$ and $\mathbf{1}_{5}$ were analyzed using the model of the coupled Gaussian/Lorentzian distance and exchange coupling distributions described above in Section 3.7.1. For compound $\mathbf{1}_{5}$, a global minimum was located already during the initial grid search using a large rough parameter grid with both Lorentzian and Gaussian distribution shapes. By reducing search ranges and applying smaller steps (i.e. by proceeding with smaller and finer parameter grids in the vicinity of global minimum found) calculations converged to the anticipated solution with narrow interspin distance distribution $\left(r_{\text {mean }} \sim 4.5 \mathrm{~nm}\right)$ and no exchange coupling. The best solution with Lorentzian distribution shapes was $r_{\text {mean }}=4.50 \mathrm{~nm}, \sigma_{r}=0.10 \mathrm{~nm}, J_{\text {mean }}=0 \mathrm{MHz}$,
$\sigma_{J}=0 \mathrm{MHz}$, whereas for Gaussian shapes we found $r_{\text {mean }}=$ $4.58 \mathrm{~nm}, \sigma_{r}=0.18 \mathrm{~nm}, J_{\text {mean }}=-0.03 \mathrm{MHz}, \sigma_{J}=0.09 \mathrm{MHz}$. Details of the grid search results are given in the ESI1. $\dagger$

Fig. 6(a) shows spin coupling spectra corresponding to the best solutions of the grid search procedure for both distribution types used. Except for the high-frequency regions of the spectra marked by arrows, we observe an excellent match between both grid search results and experimental data. Furthermore, spectra obtained from the grid search analysis nearly perfectly coincide with the spectrum corresponding to the solution obtained using regularization in the distance domain and a standard dipolar $K(t, r)$ kernel.

The most likely reason accounting for most of the aforementioned deviations in the high-frequency regions between the modelled and experimental spectra of $\mathbf{1}_{5}$ is residual orientation selection, which might be present in the measured orientation-averaged data. Indeed, for a single, relatively narrowly distributed distance one expects to observe a typical Pake-pattern like frequency spectrum with the perpendicularand the parallel-to-field singularities well seen and correctly weighted. In the case of compound $\mathbf{1}_{5}$, the position and the shape of the perpendicular-to-field features (or "horns") of the spectrum match well. The parallel-to-field features (or "wings") are underrepresented in the experimental data compared to all simulations as well as to the Tikhonov regularization fit. This indicates that these parallel orientations of the interspin axis with respect to the magnetic field were under-sampled during data acquisition.

The spin-spin coupling situation in the shorter compound $\mathbf{1}_{3}$ is more complex. The spectrum (Fig. 6(b)) is comprised of well-pronounced moderately broadened horns at about $\pm 1.75 \mathrm{MHz}$ and a very broad contribution extending beyond $\pm 12 \mathrm{MHz}$. Such a frequency content together with the very stiff nature of the spacer indicates a distribution of exchange couplings.

For Lorentzian distributions, the initial search using a large rough parameter grid revealed a global minimum. Subsequent searches with finer parameter grids around that point yield the
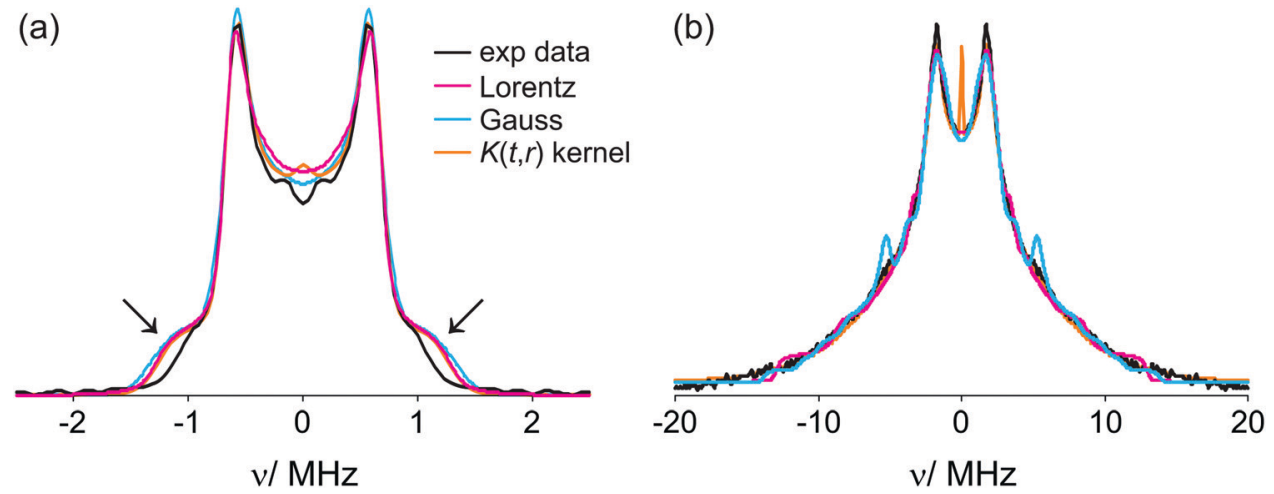

Fig. 6 Best solutions within a model of correlated exchange and dipolar coupling distributions for [Cu-PyMTA]-[Cu-PyMTA] compounds $\mathbf{1}_{5}$ (a) and $\mathbf{1}_{3}$ (b). The Gaussian distribution model is shown in cyan, the Lorentzian one in purple, experimental spectra in black and solutions obtained with the $K(t, r)$ kernel (DeerAnalysis) are shown in orange. Arrows in (a) are pointing to the high frequency region in which the experimental spectrum of $\mathbf{1}_{5}$ is affected by orientation selection. 
best-fit solution with $r_{\text {mean }}=2.53 \mathrm{~nm}, \sigma_{r}=0.09 \mathrm{~nm}, J_{\text {mean }}=$ $-5.38 \mathrm{MHz}$ and $\sigma_{J}=0.04 \mathrm{MHz}$, (Fig. 6(b)). Contrary to this, the initial search using a large rough parameter grid and Gaussian distributions revealed two solutions with similar quality (rmsd figures). Subsequent fine grid searches in the vicinity of these solutions yielded the following two results featuring somewhat higher and somewhat lower exchange coupling values: $r_{\text {mean }}=$ $2.79 \mathrm{~nm}, \sigma_{r}=0.36 \mathrm{~nm}, J_{\text {mean }}=-8.19 \mathrm{MHz}$ and $\sigma_{J}=0.1 \mathrm{MHz}$ and $r_{\text {mean }}=2.5 \mathrm{~nm}, \sigma_{r}=0.16 \mathrm{~nm}, J_{\text {mean }}=-5.5 \mathrm{MHz}, \sigma_{J}=0.08 \mathrm{MHz}$. This latter solution with shorter mean distance and smaller exchange coupling constant is very close to the result obtained using Lorentzian distributions. Details of the grid search analysis of compound $\mathbf{1}_{3}$ are given in ESI1. $\dagger$ Both solutions corresponding to the Gausssian distributions are very similar in quality to each other. However, in terms of describing the experimental data both solutions using the Gaussian distributions are worse than the solution obtained with the Lorentzian distributions that seemingly describes the experimental data in a nearly perfect way. Note that all solutions either with the Lorentzian or Gaussian distribution shapes underestimate the mean interspin distance expected from the structure of $\mathbf{1}_{3}$. This fact, together with the discussed deviations between the model solutions in terms of the interspin distance and the exchange coupling values may be attributed to the use of simplified distribution functions and the model assumption of a strict correlation between the dipole-dipole and exchange coupling distributions - which may have led to error compensation during the computations. The better fit of the experimental data with Lorentzian shaped distributions as well as the finding of a single best solution in that case indicates most probably that the underlying distributions are better described by slowly decaying tails implied by the Lorentzian shape. Nevertheless, all grid search calculations agree qualitatively in the description of the coupling situation in [Cu-PyMTA]-[Cu-PyMTA] compound $\mathbf{1}_{3}$ : a moderate ferromagnetic coupling is predicted. This prediction was subject to further analysis using the approach of regularization in the exchange-coupling domain as is discussed in the next section. Note finally that in the case of a narrow distance distribution (compound $\mathbf{1}_{5}$ ) the absence of significant exchange coupling was recognized by this fitting approach, despite the potential possibility to describe part of the couplings with distributed exchange.

\subsection{Regularization in the exchange-coupling domain}

This approach was first applied to [Cu-PyMTA]-[Cu-PyMTA] compounds $\mathbf{1}_{3}$ and $\mathbf{1}_{5}$ for which the initial characterization of the spin-spin coupling situation was described above. Based on the known structures of $\mathbf{1}_{3}$ and $\mathbf{1}_{5}$ we could make a reasonable guess on the corresponding interspin distance distributions. Generally, for [Cu-PyMTA]-[Cu-PyMTA] molecules, the estimated input distance distributions are supported by results from earlier distance measurements on the corresponding rulers with $\mathrm{Mn}$ (II) and $\mathrm{Gd}(\mathrm{III})$ as the central metal ion. ${ }^{71,110,112}$ In good agreement with the previous results, using the model of coupled distributions and the Tikhonov regularization approaches using the standard $K(t, r)$ kernel, a very narrow distribution with zero mean exchange-coupling was obtained for $\mathbf{1}_{5}$, whereas for the shorter compound $\mathbf{1}_{3}$ a relatively broad exchange coupling distribution was obtained, Fig. 7 . The shape of this distribution is largely bimodal with main peaks at zero and about $-4 \mathrm{MHz}$. This possibly points to coexistence of strained molecular structures with almost vanishing exchange
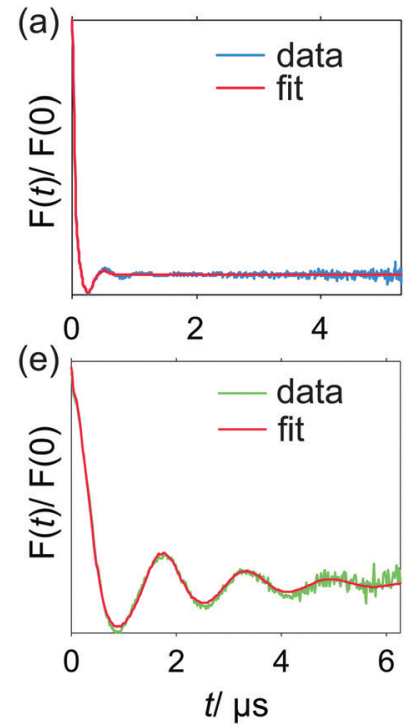
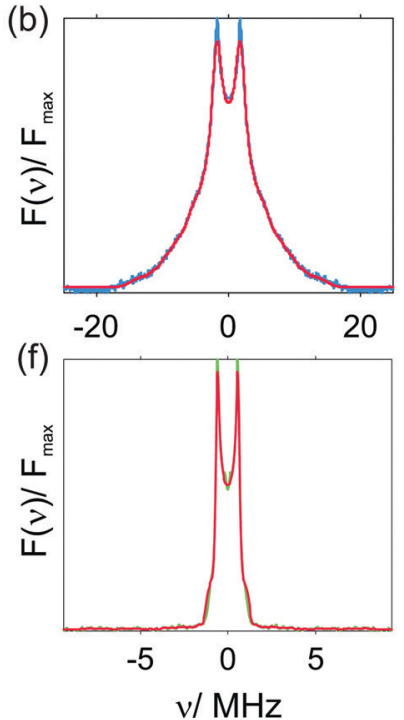
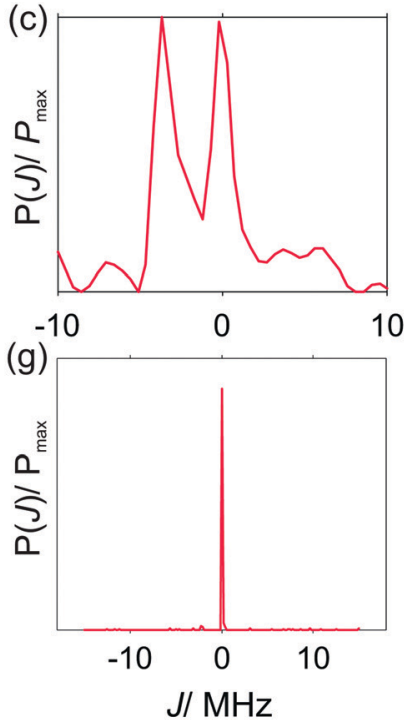
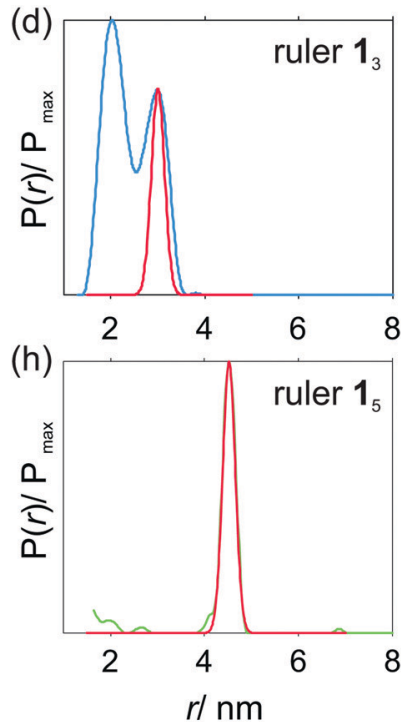

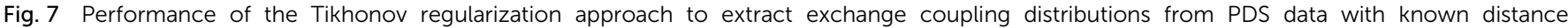

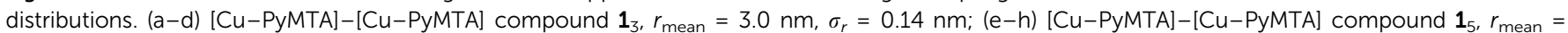

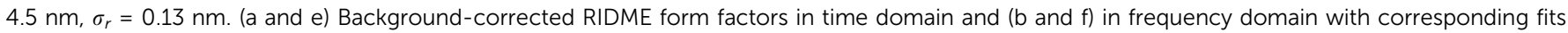

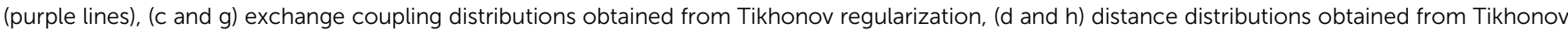

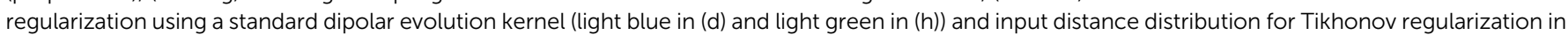
exchange-coupling domain (red lines). 
coupling as well as ones with distributed significant exchange couplings. The shape of the total exchange coupling distribution, not resembling any regular Lorentzian or Gaussian distribution, may also explain the larger coupling and shorter spin-spin distance predicted for this compound by the coupled distributions model and the better performance of Lorentzian distributions with respect to Gaussian ones. However, qualitatively, for compound $\mathbf{1}_{3}$ the results from the coupled exchange and distance distribution model match the one from regularization in the exchange-coupling domain by indicating a moderate ferromagnetic exchange coupling. Stability checks for the extracted solutions within the regularization approach are presented in ESI1. $\dagger$ Note that the assumed distribution of dipole-dipole couplings based on the molecular geometry may be somewhat imprecise because of the spatial distribution of spin density, yet, small deviations in the mean distance do not strongly affect the estimated distribution of the exchange couplings (see ESI $1 \dagger$ ).

We thus conclude that both investigated models are able to recognize the presence or absence of distributed exchange couplings and to estimate the strength of distributed exchange couplings as long as other distortions, such as orientation selection or multi-spin contributions, can be ruled out or suppressed. However, for a precise, quantitative extraction of exchange coupling parameters, precise knowledge of the spin-spin distance distribution would be required.
When the spin-spin distance distribution can be estimated by some independent method, the use of regularization in the exchange domain appears advantageous, since it is not restricting the shape of the exchange coupling distribution by an ad hoc model. Due to the uncorrelated dipolar and exchange coupling distributions, this approach is also more stable with respect to small distortions of the spin-spin coupling spectrum, which may result from orientation averaging imperfections or any other experimental artefacts.

\subsection{Neural network analysis}

We also tested the performance of two recent PDS data processing approaches for computing interspin distance distributions based on the usage of deep neural networks available in DeerAnalysis2018. Both approaches differ fundamentally from a commonly used distance extraction procedure based on the kernel inversion stabilized by a (Tikhonov) regularization. ${ }^{66}$ The first approach is based on the generic network that is designed to obtain distance distribution from primary PDS data in the absence of exchange interactions in the spin-spin pair. The second approach is based on the exchange resilient network and was developed to recover non-distorted distance distributions in the presence of exchange coupling in the primary data.

Fig. 8 shows that the generic neural network is able to capture correctly the strongly curved RIDME background in
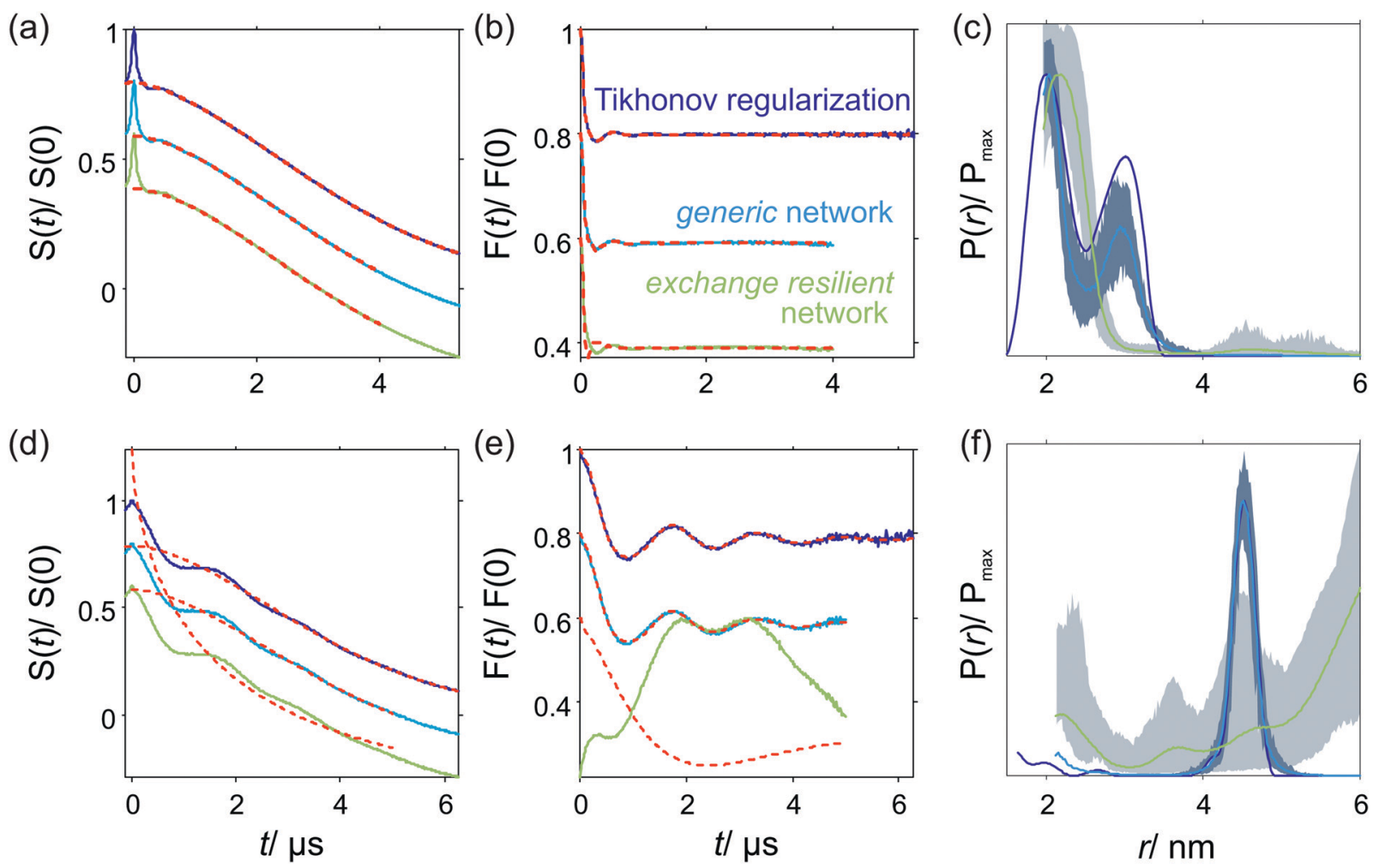

Fig. 8 Performance of deep neural networks for distance analysis in the presence and absence of exchange couplings. (a-c) [Cu-PyMTA]-[Cu-PyMTA] compound $\mathbf{1}_{3}$ - exchange coupling present; (d-f) [Cu-PyMTA]-[Cu-PyMTA] compound $\mathbf{1}_{5}$ - exchange coupling absent. (a and d) Primary RIDME data and corresponding background fit (red, dashed lines); (b and e) background-corrected form factors and corresponding fit (red, dashed lines); (c and f) resulting distance distribution and corresponding uncertainties: dark grey - generic network, light grey - exchange resilient network. 
the primary data of both rulers $\mathbf{1}_{3}$ and $\mathbf{1}_{5}$. Furthermore, the associated distance distributions match those obtained using the standard $K(t, r)$ kernel and Tikhonov regularization well: in case of ruler $\mathbf{1}_{5}$ that exhibits no exchange coupling this match is perfect, while in the case of ruler $\mathbf{1}_{3}$ in which (distributed) exchange coupling is present apparent distance distributions obtained by both methods agree reasonably well. On the contrary, the exchange resilient network failed when applied to these RIDME data. In case of the shorter compound $\mathbf{1}_{3}$, the background decay was apparently well described by the neural network analysis. However, the resulting distance distribution a single relatively broad peak at around $2.5 \mathrm{~nm}$ - strongly contradicts the interspin distance expected for this compound based on its chemical structure. In case of the longer compound $\mathbf{1}_{5}$, the background decay calculation failed altogether resulting in an unrealistic distance distribution.

Similar results were obtained when analyzing the other compounds as shown in ESI1. $\dagger$ We thus conclude that the exchange resilient network is not capable of realistically approximating the distributed exchange couplings in the presented PDS data, probably because the networks were trained with singlevalued exchange couplings. This problem may not be easy to fix by extending the training set, since the problem of simultaneous uncorrelated distribution of dipole-dipole and exchange couplings does not have a unique solution unless further restraints are introduced. Analysis of all the other compounds was therefore performed by regularization in the exchangecoupling domain with the $K(t, J)_{p(r)}$ kernel.

\subsection{Systematic analysis of reported PDS data by regularization in the exchange-coupling domain}

Results of extracting exchange couplings by regularization in the exchange-coupling domain are presented in Fig. 9 for the rulers $2_{1}, 2_{3}, 3$ and $\mathbf{4}$. The estimates of the input distance distributions needed for this approach were made on the basis of the molecular structures of the compounds (magenta distributions in Fig. 9(d, h, l and p)).

A spin pair consisting of a $\mathrm{Cu}(\mathrm{II})$ ion coupled with a nitroxide radical represented here by the [Cu-PyMTA]-nitroxide rulers $2_{n}$ $(n=1,3$, Fig. 9(a and b)) behaves very similar to the spin pair consisting of two coupled $\mathrm{Cu}(\mathrm{II})$ ions represented by the [Cu-PyMTA]-[Cu-PyMTA] rulers $\mathbf{1}_{n}(n=3,5)$ as discussed above. Primary PDS data of the long [Cu-PyMTA]-nitroxide ruler $2_{3}$ exhibit long lasting oscillations extending beyond the experimentally chosen detection window of $5 \mu$ s thus indicating that the underlying interspin distance distribution must be very narrow (Fig. 9(e)). Direct processing of these data using a standard dipolar $K(t, r)$ kernel resulted in a narrow peak centered at $3.77 \mathrm{~nm}$ matching the prediction of $3.80 \mathrm{~nm}$ based on the molecular structure. The calculation of an exchange coupling distribution in this case reveals essentially its absence, as expected (Fig. 9(c)). Primary PDS data for the shorter [Cu-PyMTA]nitroxide compound $2_{1}$ show a very strongly damped oscillation, (Fig. 9(a)). Direct processing of this trace with the $K(t, d)$ kernel expectedly results in a broad apparent distance distribution (Fig. 9(d)), being incompatible with the molecular structure.
The calculation of the exchange coupling distribution using a narrow distance peak at $2.37 \mathrm{~nm}$ (estimated on the basis of the molecular structure) yields a broad band in the exchange coupling domain with a mean coupling value of about $-2 \mathrm{MHz}$, (Fig. 9(c)).

For the [Cu-TAHA]-based rulers, the conjugated $\pi$-system of the spacer is not strongly coupled with the $\mathrm{Cu}(\mathrm{II})$ ion. It appears, however, that some exchange coupling mechanism exists for the $\mathrm{Cu}$-TAHA complex studied here. The absence of exchange interaction is nicely observed in [Cu-TAHA]-nitroxide ruler 4 (Fig. 9i-1). For this compound, structure calculation suggests a mean interspin distance of about $2.7 \mathrm{~nm}$ which is in good agreement with the experimentally obtained narrow distance peak centered at $2.64 \mathrm{~nm}$ and the extraction of exchange coupling results in the narrow coupling band centered at zero. In contrast to this, for [Cu-TAHA]-[Cu-TAHA] compound 3 our analysis indicates a fraction of strained molecular structures with negligible exchange coupling and another fraction of such structures with distributed exchange coupling of ferromagnetic type. In comparison to [Cu-PyMTA]-[Cu-PyMTA] compound $\mathbf{1}_{3}$, the fraction with significant exchange couplings is smaller and the mean exchange coupling for this fraction is slightly weaker: $\sim-2.5 \mathrm{MHz}$ for [Cu-TAHA]-[Cu-TAHA] compound 3) instead of $\sim-4 \mathrm{MHz}$ for [Cu-PyMTA]-[Cu-PyMTA] compound $\mathbf{1}_{3}$. The presence of such a non-zero mean exchange coupling in [Cu-TAHA $]-[\mathrm{Cu}-\mathrm{TAHA}]$ compound 3 indicates that there is a geometric arrangement that allows for coupling. As a speculation, this might be related to the spatial arrangement of those carboxylic groups of TAHA, which are not directly coordinated to the $\mathrm{Cu}(\mathrm{II})$ ion and facilitate through-space exchange coupling mechanism.

Finally, we should mention as an outlook that a comparison of the presented here PDS-based analysis and a more common CW EPR-based analysis of the exchange couplings in solution, ${ }^{113}$ based on the Kivelson's rotational averaging description, ${ }^{114}$ or in a frozen glassy state would be of significant interest. Similar studies in the frozen glassy state have been successfully performed to analyse systems with only throughspace dipolar couplings present. ${ }^{115}$ However, when performing such a comparison one would have to face the problem that applicability range for simultaneous use of both techniques would be rather narrow: the lower limit for the detection of spin-spin couplings in CW EPR is usually around $10 \mathrm{MHz}$ for nitroxide biradicals, which are characterized by narrower EPR lines, as compared to $\mathrm{Cu}$ (II). For the $\mathrm{Cu}$ (II) case this lower limit might get even larger, while the upper limit for detecting the spin-spin couplings with PDS is hardware dependent, and it usually lies somewhere between $50 \mathrm{MHz}$ and $100 \mathrm{MHz}$.

In addition to this, we would like to mention that in contrast to the EPR data in the frozen state, the solution EPR reports on the rotationally averaged exchange coupling. In other words, this experiment would provide a single exchange coupling value, which might make a wrong impression that also in the frozen state all biradicals are characterized by the same coupling. The low-temperature PDS data we reported here clearly show that this is an oversimplification of the actual 

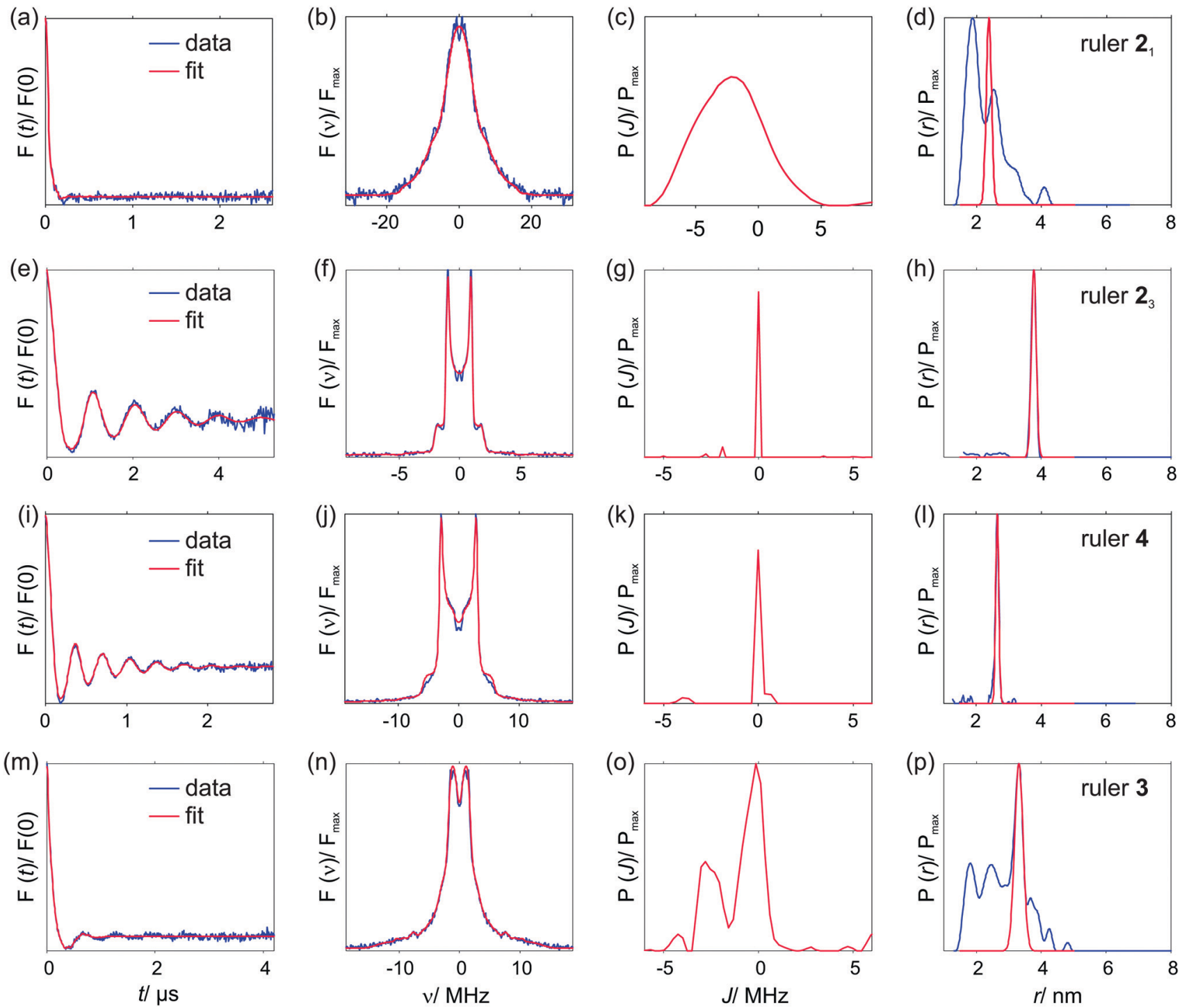

Fig. 9 Extraction of exchange couplings from PDS data by Tikhonov regularization using anticipated narrow distance distributions for (a-d) [Cu-PyMTA]nitroxide ruler $\mathbf{2}_{1},(\mathrm{e}-\mathrm{h})$ [Cu-PyMTA]-nitroxide ruler $\mathbf{2}_{3},(\mathrm{i}-\mathrm{l})[\mathrm{Cu}-\mathrm{TAHA}]-$ nitroxide ruler $\mathbf{4}$ and $(\mathrm{m}-\mathrm{p})[\mathrm{Cu}-\mathrm{TAHA}]-[\mathrm{Cu}-\mathrm{TAHA}]$ ruler $\mathbf{3}$. (a, e, i and m) Background-corrected form factor in time domain, $(b, f, j$ and $n$ ) in frequency domain with corresponding fits (magenta lines); ( $c, g, k$ and o) resulting exchange coupling distribution obtained from Tikhonov regularization; ( $d, h, l$ and p) distance distribution obtained from Tikhonov regularization using a standard dipolar evolution kernel (blue lines) and input distance distribution for exchange-coupling Tikhonov regularization (magenta lines).

situation. Unravelling how does the exchange averaging happen in solution based on the ambient temperature solution CW EPR and low temperature PDS data would be an interesting study as well, however this would imply yet another level of complexity for the experimental work and for the analysis.

\section{Conclusions}

In this work we have introduced two different approaches to evaluate distributed exchange couplings in molecular rulers. The first approach is based on fitting coupled distance and exchange coupling distributions of Lorentzian or Gaussian shape. The second approach requires the distance distribution as an input and performs Tikhonov regularization only along the exchange coupling dimension. The latter approach does not presume any particular shape of the exchange coupling distribution, but requires that the distance distribution is narrow. Both models were able to differentiate between the absence and the presence of significant exchange interaction, to determine the exchange coupling regime (ferro- or antiferromagnetic) and to estimate its overall shape. If some knowledge on the distance distribution is available, the Tikhonov regularization in the exchange-coupling domain approach is found to better describe the underlying exchange coupling distribution. Our analysis revealed that exchange couplings may be present in the studied compounds with $\pi$-conjugated spacers up to at least $3.3 \mathrm{~nm}$ spinspin distance, while it is negligible for distances $r \geq 4.5 \mathrm{~nm}$ in $\mathrm{Cu}$ (II)-Cu(II) and for $r \geq 3.8 \mathrm{~nm}$ in $\mathrm{Cu}$ (II)-nitroxide systems. Interestingly, while the disruption of the coupling between 
$\mathrm{Cu}$ (II) ion and $\pi$-conjugated system of the effective spacer in the case of TAHA-ligand leads to the absence of distributed exchange couplings at least for distances $r \geq 2.6 \mathrm{~nm}$ in [Cu-TAHA]-nitroxide ruler $\mathbf{4}$, for the [Cu-TAHA]-[Cu-TAHA] ruler 3, with an even slightly longer spin-spin distance, nonzero exchange coupling is observed.

Analysis performed with DEERNet neural networks, as available in DeerAnalysis2018, was able to recover the strongly curved background in RIDME experiments using the generic model. However, within the exchange resilient model the neural network analysis was not able to identify the exchange couplings and provide the correct distance distribution for our data, likely because the present form of approximating exchange by a single $J$ value in DEERNet appears to be unable to treat such cases with broadly distributed exchange couplings. Our work shows that approaches to determine exchange distributions in stiff synthetic molecules most likely require that the distance distribution and thus the distribution of dipolar couplings is known. Provided that the molecular geometry is known and the spin density distributions at both spin centres can be estimated, it should be possible to compute the dipole-dipole contribution independently and thus to determine the distribution of exchange couplings, e.g. by the here presented one-dimensional Tikhonov regularization.

\section{Conflicts of interest}

Authors declare no conflicts of interest.

\section{Acknowledgements}

The work was supported by the Swiss National Science Foundation (grant 200020_169057) and by the Deutsche Forschungsgemeinschaft (DFG) within SPP 1601 (GO555/6-2).

\section{References}

1 T. Prisner, M. Rohrer and F. MacMillan, Pulsed EPR Spectroscopy: Biological Applications, Annu. Rev. Phys. Chem., 2001, 52, 279-313.

2 A. Schweiger and G. Jeschke, Principles of Pulse Electron Paramagnetic Resonance, Oxford University Press, 2001.

3 A. Bencini and D. Gatteschi, EPR of Exchange Coupled Systems, Springer Verlag, Berlin, 1990.

4 O. Schiemann and T. F. Prisner, Long-range distance determinations in biomacromolecules by EPR spectroscopy, Q. Rev. Biophys., 2007, 40, 1-53.

5 G. Jeschke, DEER distance measurements on proteins, Annu. Rev. Phys. Chem., 2012, 63, 419-446.

6 I. Krstic, B. Endeward, D. Margraf, A. Marko and T. F. Prisner, Structure and Dynamics of Nucleic Acids, Top. Curr. Chem., 2012, 321, 159-198.

7 A. Caneschi, D. Gatteschi, R. Sessoli and P. Rey, Toward Molecular Magnets: The Metal-Radical Approach, Acc. Chem. Res., 1989, 392-398.
8 S. Gao and M. Affronte, Molecular nanomagnets and related phenomena, Springer, 2015, vol. 164.

9 A. S. L. Thankamony, J. J. Wittmann, M. Kaushik and B. Corzilius, Dynamic nuclear polarization for sensitivity enhancement in modern solid-state NMR, Prog. Nucl. Magn. Reson. Spectrosc., 2017, 102-103, 120-195.

10 P. Caravan, J. J. Ellison, T. J. McMurry and R. B. Lauffer, Gadolinium(III) Chelates as MRI Contrast Agents: Structure, Dynamics, and Applications, Chem. Rev., 1999, 99, 2293-2352.

11 S. Aime, M. Botta, M. Fasano and E. Terreno, Lanthanide (III) chelates for NMR biomedical applications, Chem. Soc. Rev., 1998, 27, 19-29.

12 A. S. Merbach, The chemistry of contrast agents in medical magnetic resonance imaging, John Wiley \& Sons, 2013.

13 L. Helm, Optimization of gadolinium-based MRI contrast agents for high magnetic-field applications, Future Med. Chem., 2010, 2, 385-396.

14 R. E. Winpenny, Quantum information processing using molecular nanomagnets as qubits, Angew. Chem., Int. Ed., 2008, 47, 7992-7994.

15 G. Arom, D. Aguila, P. Gamez, F. Luis and O. Roubeau, Design of magnetic coordination complexes for quantum computing, Chem. Soc. Rev., 2012, 41, 537-546.

16 J. M. Clemente-Juan, E. Coronado and A. Gaita-Ariño, Magnetic polyoxometalates: from molecular magnetism to molecular spintronics and quantum computing, Chem. Soc. Rev., 2012, 41, 7464-7478.

17 R. Meng, J. Becker, F.-T. Lin, S. Saxena and S. G. Weber, Binding of copper(II) to thyrotropin-releasing hormone (TRH) and its analogs, Inorg. Chim. Acta, 2005, 358, 2933-2942.

18 C. W. M. Kay, H. El Mkami, R. Cammack and R. W. Evans, Pulsed ELDOR Determination of the Intramolecular Distance between the Metal Binding Sites in Dicupric Human Serum Transferrin and Lactoferrin, J. Am. Chem. Soc., 2007, 129, 4868-4869.

19 B.-K. Shin and S. Saxena, Direct Evidence That All Three Histidine Residues Coordinate to Cu(II) in Amyloid- $\beta 1-16$, Biochemistry, 2008, 47, 9117-9123.

20 K. J. Waldron, J. C. Rutherford, D. Ford and N. J. Robinson, Metalloproteins and metal sensing, Nature, 2009, 460, 823.

21 A. Lewin, J. P. Hill, R. Boetzel, T. Georgiou, R. James, C. Kleanthous and G. R. Moore, Site-specific labeling of proteins with cyclen-bound transition metal ions, Inorg. Chim. Acta, 2002, 331, 123-130.

22 T. F. Cunningham, M. D. Shannon, M. R. Putterman, R. J. Arachchige, I. Sengupta, M. Gao, C. P. Jaroniec and S. Saxena, Cysteine-Specific Cu2+ Chelating Tags Used as Paramagnetic Probes in Double Electron Electron Resonance, J. Phys. Chem. B, 2015, 119, 2839-2843.

23 Z. Yang, M. Ji, T. F. Cunningham and S. Saxena, Chapter Seventeen $-\mathrm{Cu}^{2+}$ as an ESR Probe of Protein Structure and Function, in Methods in Enzymology, ed. P. Z. Qin and K. Warncke, Academic Press, 2015, vol. 563, pp. 459-481.

24 G. E. Fanucci and D. S. Cafiso, Recent advances and applications of site-directed spin labeling, Curr. Opin. Struct. Biol., 2006, 16, 644-653. 
25 J. P. Klare and H.-J. Steinhoff, Spin labeling EPR, Photosynth. Res., 2009, 102, 377-390.

26 E. Bordignon, Site-directed spin labeling of membrane proteins, Top. Curr. Chem., 2012, 321, 121-157.

27 W. L. Hubbell, C. J. Lopez, C. Altenbach and Z. Yang, Technological advances in site-directed spin labeling of proteins, Curr. Opin. Struct. Biol., 2013, 23, 725-733.

28 J. L. Wort, K. Ackermann, A. Giannoulis, A. J. Stewart, D. G. Norman and B. E. Bode, Sub-Micromolar Pulse Dipolar EPR Spectroscopy Reveals Increasing CuIIlabelling of Double-Histidine Motifs with Lower Temperature, Angew. Chem., Int. Ed., 2019, 58, 11681-11685.

29 D. M. Engelhard, A. Meyer, A. Berndhäuser, O. Schiemann and G. H. Clever, Di-Copper (II) DNA G-Quadruplexes as EPR Distance Rulers, Chem. Commun., 2018, 54, 7455-7458.

30 A. Gamble Jarvi, K. Ranguelova, S. Ghosh, R. T. Weber and S. Saxena, On the Use of Q-Band Double Electronâ, Electron Resonance To Resolve the Relative Orientations of Two Double Histidine-Bound $\mathrm{Cu} 2+$ Ions in a Protein, J. Phys. Chem. B, 2018, 122, 10669-10677. PMID: 30372072.

31 S. Ghosh, V. Garcia, K. Singewald, S. M. Damo and S. Saxena, Cu(II) EPR Reveals Two Distinct Binding Sites and Oligomerization of Innate Immune Protein Calgranulin C, Appl. Magn. Reson., 2018, 49, 1299-1311.

32 S. Ghosh, S. Saxena and G. Jeschke, Rotamer Modelling of $\mathrm{Cu}(\mathrm{II})$ Spin Labels Based on the Double-Histidine Motif, Appl. Magn. Reson., 2018, 49, 1281-1298.

33 F. D. Breitgoff, K. Keller, M. Qi, D. Klose, M. Yulikov, A. Godt and G. Jeschke, UWB DEER and RIDME distance measurements in $\mathrm{Cu}(\mathrm{II})-\mathrm{Cu}(\mathrm{II})$ spin pairs, J. Magn. Reson., 2019, 106560.

34 M. Asano-Someda, N. Toyama and Y. Kaizu, Weak exchange interaction of copper(II) pairs in rigidly linked porphyrin dimers, Mol. Cryst. Liq. Cryst., 2002, 379, 165-170.

35 N. Toyama, M. Asano-Someda and Y. Kaizu, EPR spectra of gable-type copper(II) porphyrin dimers in fluid solution: extraction of exchange interaction in weakly coupled doublet pairs, Mol. Phys., 2003, 101, 733-742.

36 B. E. Bode, J. Plackmeyer, T. F. Prisner and O. Schiemann, PELDOR measurements on a nitroxide-labeled $\mathrm{Cu}(\mathrm{II})$ porphyrin: Orientation selection, spin-density distribution, and conformational flexibility, J. Phys. Chem. A, 2008, 112, 5064-5073.

37 B. E. Bode, J. Plackmeyer, M. Bolte, T. F. Prisner and O. Schiemann, PELDOR on an exchange coupled nitroxide copper(II) spin pair, J. Organomet. Chem., 2009, 694, 1172-1179.

38 S. Richert, J. Cremers, I. Kuprov, M. D. Peeks, H. L. Anderson and C. R. Timmel, Constructive quantum interference in a bis-copper six-porphyrin nanoring, Nat. Commun., 2017, 8, 14842.

39 S. Richert, I. Kuprov, M. D. Peeks, E. A. Suturina, J. Cremers, H. L. Anderson and C. R. Timmel, Quantifying the exchange coupling in linear copper porphyrin oligomers, Phys. Chem. Chem. Phys., 2017, 19, 16057-16061.
40 D. Gatteschi, A. L. Barra, A. Caneschi, A. Cornia, R. Sessoli and L. Sorace, EPR of molecular nanomagnets, Coord. Chem. Rev., 2006, 250, 1514-1529.

41 J. van Slageren, New Directions in Electron Paramagnetic Resonance Spectroscopy on Molecular Nanomagnets, in EPR Spectroscopy: Applications in Chemistry and Biology, ed. M. Drescher and G. Jeschke, Springer Berlin Heidelberg, Berlin, Heidelberg, 2012, pp. 199-234.

42 S. S. Eaton, K. M. More, B. M. Sawant and G. R. Eaton, Use of the ESR half-field transition to determine the interspin distance and the orientation of the interspin vector in systems with two unpaired electrons, J. Am. Chem. Soc., 1983, 105, 6560-6567.

43 S. A. Dzuba, P. Gast and A. J. Hoff, ESEEM study of spinspin interactions in spin-polarised $\mathrm{P}+\mathrm{QA}-$ pairs in the photosynthetic purple bacterium rhodobacter sphaeroides R26, Chem. Phys. Lett., 1995, 236, 595-602.

44 G. Jeschke, Determination of the Nanostructure of Polymer Materials by Electron Paramagnetic Resonance Spectroscopy, Macromol. Rapid Commun., 2002, 23, 227-246.

45 P. Wautelet, A. Bieber, P. Turek, J. L. Moigne and J.-J. André, Magnetic properties of iminonitroxide and nitronylnitroxide based diradicals, Mol. Cryst. Liq. Cryst. Sci. Technol., Sect. A, 1997, 305, 55-67.

46 K. M. More, G. R. Eaton and S. S. Eaton, Metal-nitroxyl interactions. 47. epr spectra of two-spin-labeled derivatives of edta coordinated to paramagnetic metal ions, Inorg. Chem., 1986, 25, 2638-2646.

47 T. Smith and J. Pilbrow, The determination of structural properties of dimeric transition metal ion complexes from EPR spectra, Coord. Chem. Rev., 1974, 13, 173-278.

48 R. Calvo, EPR measurements of weak exchange interactions coupling unpaired spins in model compounds, Appl. Magn. Reson., 2007, 31, 271.

49 S. S. Eaton, G. R. Eaton and C. Chang, Synthesis and geometry determination of cofacial diporphyrins. EPR spectroscopy of dicopper diporphyrins in frozen solution, J. Am. Chem. Soc., 1985, 107, 3177-3184.

50 R. G. Larsen and D. J. Singel, Double electron-electron resonance spin-echo modulation: Spectroscopic measurement of electron spin pair separations in orientationally disordered solids, J. Chem. Phys., 1993, 98, 5134-5146.

51 A. I. Kokorin, Regularities of the spin exchange coupling through a bridge in nitroxide biradicals, Appl. Magn. Reson., 2004, 26, 253-274.

52 G. Jeschke, M. Sajid, M. Schulte, N. Ramezanian, A. Volkov, H. Zimmermann and A. Godt, Flexibility of shape-persistent molecular building blocks composed of p-phenylene and ethynylene units, J. Am. Chem. Soc., 2010, 132, 10107-10117.

53 A. Godt, M. Schulte, H. Zimmermann and G. Jeschke, How flexible are poly(para-phenyleneethynylene)s?, Angew. Chem., Int. Ed., 2006, 118, 7722-7726.

54 A. M. Bowen, C. E. Tait, C. R. Timmel and J. R. Harmer, Structural Information from Spin-Labels and Intrinsic Paramagnetic Centres in the Biosciences, Springer Berlin Heidelberg, Berlin, Heidelberg, 2013, pp. 283-327. 
55 D. Abdullin, N. Florin, G. Hagelueken and O. Schiemann, EPR-Based Approach for the Localization of Paramagnetic Metal Ions in Biomolecules, Angew. Chem., Int. Ed., 2015, 127, 1847-1851.

56 I. Ritsch, H. Hintz, G. Jeschke, A. Godt and M. Yulikov, Improving the accuracy of $\mathrm{Cu}(\mathrm{II})$-nitroxide $\mathrm{RIDME}$ in the presence of orientation correlation in water-soluble Cu(II)-nitroxide rulers, Phys. Chem. Chem. Phys., 2019, 21, 9810-9830.

57 A. Milov, A. Maryasov and Y. D. Tsvetkov, Pulsed electron double resonance (PELDOR) and its applications in freeradicals research, Appl. Magn. Reson., 1998, 15, 107-143.

58 A. Maryasov, Y. D. Tsvetkov and J. Raap, Weakly coupled radical pairs in solids: ELDOR in ESE structure studies, Appl. Magn. Reson., 1998, 14, 101-113.

59 A. Weber, O. Schiemann, B. Bode and T. F. Prisner, PELDOR at $\mathrm{S}$-and $\mathrm{X}$-band frequencies and the separation of exchange coupling from dipolar coupling, J. Magn. Reson., 2002, 157, 277-285.

60 G. W. Reginsson, R. I. Hunter, P. A. Cruickshank, D. R. Bolton, S. T. Sigurdsson, G. M. Smith and O. Schiemann, W-band PELDOR with $1 \mathrm{~kW}$ microwave power: Molecular geometry, flexibility and exchange coupling, J. Magn. Reson., 2012, 216, 175-182.

61 D. Margraf, P. Cekan, T. Prisner, S. T. Sigurdsson and O. Schiemann, Ferro-and antiferromagnetic exchange coupling constants in peldor spectra, Phys. Chem. Chem. Phys., 2009, 11, 6708-6714.

62 L. Kulik, S. Dzuba, I. Grigoryev and Y. Tsvetkov, Electron dipole-dipole interaction in ESEEM of nitroxide biradicals, Chem. Phys. Lett., 2001, 343, 315-324.

63 S. Milikisyants, F. Scarpelli, M. G. Finiguerra, M. Ubbink and M. Huber, A pulsed EPR method to determine distances between paramagnetic centers with strong spectral anisotropy and radicals: The dead-time free RIDME sequence, J. Magn. Reson., 2009, 201, 48-56.

64 A. D. Milov, K. M. Salikhov and M. D. Shirov, Application of ELDOR in Electron-Spin Echo for Investigation of Paramagnetic Center Space Distribution in Solids, Fiz. Tverd. Tela, 1981, 957-982.

65 M. Pannier, S. Veit, A. Godt, G. Jeschke and H. Spiess, DeadTime Free Measurement of Dipole-Dipole Interactions between Electron Spins, J. Magn. Reson., 2000, 142, 331-340.

66 S. G. Worswick, J. A. Spencer, G. Jeschke and I. Kuprov, Deep neural network processing of DEER data, Sci. Adv., 2018, 4, eaat5218, DOI: 10.1126/sciadv.aat5218.

67 E. Narr, A. Godt and G. Jeschke, Selective Measurements of a Nitroxide-Nitroxide Separation of $5 \mathrm{~nm}$ and a NitroxideCopper Separation of $2.5 \mathrm{~nm}$ in a Terpyridine-Based Copper(II) Complex by Pulse EPR Spectroscopy, Angew. Chem., Int. Ed., 2002, 41, 3907-3910.

68 Z. Yang, J. Becker and S. Saxena, On Cu(II)-Cu(II) distance measurements using pulsed electron electron double resonance, J. Magn. Reson., 2007, 188, 337-343.

69 J. E. Lovett, A. M. Bowen, C. R. Timmel, M. W. Jones, J. R. Dilworth, D. Caprotti, S. G. Bell, L. L. Wong and
J. Harmer, Structural information from orientationally selective DEER spectroscopy, Phys. Chem. Chem. Phys., 2009, 11, 6840-6848.

70 Z. Yang, D. Kise and S. Saxena, An Approach towards the Measurement of Nanometer Range Distances Based on Cu2+ Ions and ESR, J. Phys. Chem. B, 2010, 114, 6165-6174.

71 A. Doll, M. Qi, N. Wili, S. Pribitzer, A. Godt and G. Jeschke, Gd(III)-Gd(III) distance measurements with chirp pump pulses, J. Magn. Reson., 2015, 259, 153-162.

72 S. Razzaghi, M. Qi, A. I. Nalepa, A. Godt, G. Jeschke, A. Savitsky and M. Yulikov, RIDME Spectroscopy with Gd(III) Centers, J. Phys. Chem. Lett., 2014, 5, 3970-3975.

73 A. Savitsky, A. A. Dubinskii, M. Flores, W. Lubitz and K. Mobius, Orientation-resolving pulsed electron dipolar high-field EPR spectroscopy on disordered solids: I. Structure of spin-correlated radical pairs in bacterial photosynthetic reaction centers, J. Phys. Chem. B, 2007, 111, 6245-6262.

74 K. Keller, M. Qi, C. Gmeiner, I. Ritsch, A. Godt, G. Jeschke, A. Savitsky and M. Yulikov, Intermolecular background decay in RIDME experiments, Phys. Chem. Chem. Phys., 2019, 21, 8228-8245.

$75 \mathrm{~W}$. W. Xie and P. R. Tremaine, Thermodynamics of Aqueous Diethylenetriaminepentaacetic Acid (DTPA) Systems: Apparent and Partial Molar Heat Capacities and Volumes of Aqueous $\mathrm{H}_{2} \mathrm{DTPA}^{3-}$, $\mathrm{DTPA}^{5-}$, $\mathrm{CuDTPA}^{3-}$, and $\mathrm{Cu}_{2} \mathrm{DTPA}^{-}$from 10 to $55{ }^{\circ} \mathrm{C}$, J. Solution Chem., 1999, 28, 291-325.

76 A. Doll and G. Jeschke, Fourier-transform electron spin resonance with bandwidth-compensated chirp pulses, J. Magn. Reson., 2014, 246, 18-26.

77 A. Doll and G. Jeschke, EPR-correlated dipolar spectroscopy by Q-band chirp SIFTER, Phys. Chem. Chem. Phys., 2016, 18, 23111-23120.

78 R. Tschaggelar, B. Kasumaj, M. G. Santangelo, J. Forrer, P. Leger, H. Dube, F. Diederich, J. Harmer, R. Schuhmann, I. Garca-Rubio and G. Jeschke, Cryogenic $35 \mathrm{GHz}$ pulse ENDOR probehead accommodating large sample sizes: Performance and applications, J. Magn. Reson., 2009, 200, 81-87.

79 Y. Polyhach, E. Bordignon, R. Tschaggelar, S. Gandra, A. Godt and G. Jeschke, High sensitivity and versatility of the DEER experiment on nitroxide radical pairs at Q-band frequencies, Phys. Chem. Chem. Phys., 2012, 14, 10762-10773.

80 R. Tschaggelar, F. D. Breitgoff, O. Oberhänsli, M. Qi, A. Godt and G. Jeschke, High-Bandwidth Q-Band EPR Resonators, Appl. Magn. Reson., 2017, 48, 1273-1300.

81 K. Keller, A. Doll, M. Qi, A. Godt, G. Jeschke and M. Yulikov, Averaging of nuclear modulation artefacts in RIDME experiments, J. Magn. Reson., 2016, 272, 108-113.

82 A. Tann and M. Garwood, et al., Improved performance of frequency-swept pulses using offset-independent adiabaticity, J. Magn. Reson., Ser. A, 1996, 120, 133-137.

83 A. Doll and G. Jeschke, Wideband frequency-swept excitation in pulsed EPR spectroscopy, J. Magn. Reson., 2017, 280, 46-62. 
84 S. Stoll and A. Schweiger, EasySpin, a comprehensive software package for spectral simulation and analysis in EPR, J. Magn. Reson., 2006, 178, 42-55.

85 W. Froncisz and J. S. Hyde, Broadening by strains of lines in the $g$-parallel region of $\mathrm{Cu}^{2+}$ EPR spectra, J. Chem. Phys., 1980, 73, 3123-3131.

86 F. Neese, The ORCA program system, Wiley Interdiscip. Rev.: Comput. Mol. Sci., 2011, 2, 73-78.

87 C. Lee, W. Yang and R. G. Parr, Development of the ColleSalvetti correlation-energy formula into a functional of the electron density, Phys. Rev. B: Condens. Matter Mater. Phys., 1988, 37, 785-789.

88 A. D. Becke, Density-functional thermochemistry. III. The role of exact exchange, J. Chem. Phys., 1993, 98, 5648-5652.

89 P. J. Stephens, F. J. Devlin, C. F. Chabalowski and M. J. Frisch, $A b$ Initio Calculation of Vibrational Absorption and Circular Dichroism Spectra Using Density Functional Force Fields, J. Phys. Chem., 1994, 98, 11623.

90 S. Grimme, J. Antony, S. Ehrlich and H. Krieg, A consistent and accurate $a b$ initio parametrization of density functional dispersion correction (DFT-D) for the 94 elements $\mathrm{H}-\mathrm{Pu}$, J. Chem. Phys., 2010, 132, 154104.

91 S. Grimme, S. Ehrlich and L. Goerigk, Effect of the damping function in dispersion corrected density functional theory, J. Comput. Chem., 2011, 32, 1456-1465.

92 F. Weigend and R. Ahlrichs, Balanced basis sets of split valence, triple zeta valence and quadruple zeta valence quality for $\mathrm{H}$ to $\mathrm{Rn}$ : Design and assessment of accuracy, Phys. Chem. Chem. Phys., 2005, 7, 3297-3305.

93 J. Zheng, X. Xu and D. G. Truhlar, Minimally augmented Karlsruhe basis sets, Theor. Chem. Acc., 2011, 128, 295-305.

94 C. Adamo and V. Barone, Toward reliable density functional methods without adjustable parameters: The PBE0 model, J. Chem. Phys., 1999, 110, 6158-6170.

95 F. Neese, Prediction and interpretation of the 57Fe isomer shift in Mössbauer spectra by density functional theory, Inorg. Chim. Acta, 2002, 337, 181-192.

96 D. P. Chong, Recent Advances in Density Functional Methods: (Part I), World Scientific, 1995, vol. 1.

97 G. Jeschke, V. Chechik, P. Ionita, A. Godt, H. Zimmermann, J. Banham, C. R. Timmel, D. Hilger and H. Jung, DeerAnalysis2006-a comprehensive software package for analyzing pulsed ELDOR data, Appl. Magn. Reson., 2006, 30, 473-498, http://www.epr.ethz.ch/software.html.

98 M. F. Czar, F. D. Breitgoff, D. Sahoo, M. Sajid, N. Ramezanian, Y. Polyhach, G. Jeschke, A. Godt and R. Zenobi, Linear and Kinked Oligo(phenyleneethynylene)s as Ideal Molecular Calibrants for Förster Resonance Energy Transfer, J. Phys. Chem. Lett., 2019, 10, 6942-6947.

99 M. Qi, M. Hülsmann and A. Godt, Spacers for Geometrically Well-Defined Water-Soluble Molecular Rulers and Their Application, J. Org. Chem., 2016, 81, 2549-2571.

100 D. Sahoo, S. Thiele, M. Schulte, N. Ramezanian and A. Godt, Polar tagging in the synthesis of monodisperse oligo(p-phenyleneethynylene)s and an update on the synthesis of oligoPPEs, Beilstein J. Org. Chem., 2010, 6, DOI: $10.3762 /$ bjoc.6.57.

101 S. Burnet, M.-H. Choi, P. S. Donnelly, J. M. Harrowfield, I. Ivanova, S.-H. Jeong, Y. Kim, M. Mocerino, B. W. Skelton, A. H. White, C. C. Williams and Z.-L. Zeng, Functionalization of Cage Amines with Pendant Aromatic and Heteroaromatic Substituents, Eur. J. Inorg. Chem., 2001, 1869-1881.

102 A. Godt, C. Franzen, S. Veit, V. Enkelmann, M. Pannier and G. Jeschke, Epr probes with well-defined, long distances between two or three unpaired electrons, J. Org. Chem., 2000, 65, 7575-7582.

103 M. Qi, M. Hülsmann and A. Godt, Synthesis and Hydrolysis of 4-Chloro-PyMTA and 4-Iodo-PyMTA Esters and Their Oxidative Degradation with $\mathrm{Cu}(\mathrm{I} / \mathrm{II})$ and Oxygen, Synthesis, 2016, 3773-3784.

104 A. G. Maryasov and Y. D. Tsvetkov, Formation of the pulsed electron-electron double resonance signal in the case of a finite amplitude of microwave fields, Appl. Magn. Reson., 2000, 18, 583-605.

105 J. Peisach and W. Blumberg, Structural implications derived from the analysis of electron paramagnetic resonance spectra of natural and artificial copper proteins, Arch. Biochem. Biophys., 1974, 165, 691-708.

106 X. Bogetti, S. Ghosh, A. Gamble Jarvi, J. Wang and S. Saxena, Molecular Dynamics Simulations Based on Newly Developed Force Field Parameters for $\mathrm{Cu}^{2+}$ Spin Labels Provide Insights into Double-Histidine-Based Double Electron-Electron Resonance, J. Phys. Chem. B, 2020, 124, 2788-2797. PMID: 32181671.

107 J. L. Bredas, R. Silbey, D. S. Boudreaux and R. R. Chance, Chain-length dependence of electronic and electrochemical properties of conjugated systems: polyacetylene, polyphenylene, polythiophene, and polypyrrole, J. Am. Chem. Soc., 1983, 105, 6555-6559.

108 A. M. Boldi, J. Anthony, V. Gramlich, C. B. Knobler, C. Boudon, J.-P. Gisselbrecht, M. Gross and F. Diederich, Acyclic Tetraethynylethene Molecular Scaffolding: Multinanometer-sized linearly conjugated rods with the poly(triacetylene) backbone and cross-conjugated expanded dendralenes, Helv. Chim. Acta, 1995, 78, 779-796.

109 H. Meier, J. Gerold, H. Kolshorn and B. Mühling, Extension of Conjugation Leading to Bathochromic or Hypsochromic Effects in OPV Series, Chem. - Eur. J., 2004, 10, 360-370.

110 A. Dalaloyan, M. Qi, S. Ruthstein, S. Vega, A. Godt, A. Feintuch and D. Goldfarb, EPR Distance Measurements - the Range of Accessible Distances and the Impact of Zero Field Splitting, Phys. Chem. Chem. Phys., 2015, 17, 18464-18476.

111 G. E. McDuffie, R. G. Quinn and T. A. Litovitz, Dielectric Properties of Glycerol-Water Mixtures, J. Chem. Phys., 1962, 37, 239-242.

112 K. Keller, M. Zalibera, M. Qi, V. Koch, J. Wegner, H. Hintz, A. Godt, G. Jeschke, A. Savitsky and M. Yulikov, EPR Characterization of $\mathrm{Mn}(\mathrm{II})$ Complexes for Distance 
Determination with Pulsed Dipolar Spectroscopy, Phys. Chem. Chem. Phys., 2016, 18, 25120-25135.

113 R. Wang, A. M. Brugh, J. Rawson, M. J. Therien and M. D. E. Forbes, Alkyne-Bridged Multi[Copper(II) Porphyrin] Structures: Nuances of Orbital Symmetry in Long-Range, Through-Bond Mediated, Isotropic Spin Exchange Interactions, J. Am. Chem. Soc., 2017, 139, 9759-9762. PMID: 28578583.
114 R. Wilson and D. Kivelson, ESR Linewidths in Solution. I. Experiments on Anisotropic and Spin-Rotational Effects, J. Chem. Phys., 1966, 44, 154-168.

115 J. Banham, C. Baker, S. Ceola, I. Day, G. Grant, E. Groenen, C. Rodgers, G. Jeschke and C. Timmel, Distance measurements in the borderline region of applicability of CW EPR and DEER: A model study on a homologous series of spin-labelled peptides, J. Magn. Reson., 2008, 191, 202-218. 